\title{
An adjustable robust optimization approach to scheduling of continuous industrial processes providing interruptible load
}

\author{
Qi Zhang ${ }^{\mathrm{a}}$, Michael F. Morari ${ }^{\mathrm{b}}$, Ignacio E. Grossmann ${ }^{\mathrm{a}, *}$, Arul \\ Sundaramoorthy ${ }^{\mathrm{c}}$, Jose M. Pinto ${ }^{\mathrm{d}}$ \\ ${ }^{a}$ Center for Advanced Process Decision-making, Department of Chemical Engineering, \\ Carnegie Mellon University, Pittsburgh, PA 15213, USA \\ ${ }^{b}$ Department of Chemical and Bioengineering, Swiss Federal Institute of Technology Zurich \\ (ETHZ), 8092 Zurich, Switzerland \\ ${ }^{c}$ Praxair, Inc., Business and Supply Chain Optimization RED, Tonawanda, NY 14150, \\ USA \\ ${ }^{d}$ Praxair, Inc., Business and Supply Chain Optimization RESD, Danbury, CT 06810, USA
}

\begin{abstract}
To ensure the stability of the power grid, backup capacities are called upon when electricity supply does not meet demand due to unexpected changes in the grid. As part of the demand response efforts in recent years, large electricity consumers are encouraged by financial incentives to provide such operating reserve in the form of load reduction capacities (interruptible load). However, a major challenge lies in the uncertainty that one does not know in advance when load reduction will be requested. In this work, we develop a scheduling model for continuous industrial processes providing interruptible load. An adjustable robust optimization approach, which incorporates recourse decisions using linear decision rules, is applied to model the uncertainty. The proposed model is applied to an illustrative example as well as a real-world air separation case. The results show the benefits from selling interruptible load and the value of considering recourse in the decision-making.
\end{abstract}

Keywords: Production scheduling, demand response, interruptible load, adjustable robust optimization, mixed-integer linear programming

\section{Introduction}

With rising electricity demand, deregulated electricity markets, and increasing penetration of intermittent renewable energy into the power supply mix, the level of uncertainty in the power grid has increased tremendously. As a

\footnotetext{
${ }^{*}$ Corresponding author

Email address: grossmann@cmu.edu (Ignacio E. Grossmann)
} 
result, the stable and reliable operation of the power grid has become increasingly challenging. In recent years, the notion of a smart grid has been evolving, which represents the idea of effectively coordinating the major grid operationselectricity generation, transmission, distribution, and consumption-through improved communication, holistic optimization, and market design.

One main element of the smart grid concept is the utilization of the flexibility for load adjustment on the electricity consumers' side, which is also referred to as demand response (DR). In DR, one distinguishes between dispatchable and nondispatchable DR (FERC, 2010). Dispatchable DR refers to load adjustment capacities that consumers provide to the grid operator such that these capacities can be dispatched to maintain grid stability or in times of emergency. The grid operator has control over dispatchable DR resources by either direct load control or by sending load adjustment requests to the consumers. In nondispatchable DR, consumers are not obliged to meet any load change requests by the grid operator, but rather choose to adjust their power consumption profiles based on price signals from the electricity market.

Only recently, researchers and practitioners have acknowledged the high potential benefits of demand side management (DSM), which involves energy efficiency and DR measures, for the chemical processing industry (Paulus and Borggrefe, 2011; Samad and Kiliccote, 2012; Merkert et al., 2014). In particular, the scheduling of power-intensive industrial processes has evolved into a major research field. In this context, processes such as steelmaking (Ashok, 2006; Castro et al., 2013), electrolysis (Babu and Ashok, 2008), cement production (Castro et al., 2011), and air separation (Ierapetritou et al., 2002; Karwan and Keblis, 2007; Mitra et al., 2012; Zhang et al., 2016) have been considered. In their recent review, Zhang and Grossmann (2015) present a comprehensive overview of the advances made in planning and scheduling for industrial DSM, and highlight future challenges in this area. One of the conclusions of the review is that most existing works only consider nondispatchable DR; scheduling models involving dispatchable DR are extremely scarce, mainly due to the challenge of accounting for the inherent uncertainty in the problems.

Interruptible load is a dispatchable DR resource. The power grid is designed to match electricity supply and demand at all times. When real-time electricity supply falls below the demand, e.g. due to generator failures or sudden load changes, backup capacities are called upon in order to eliminate the supplydemand gap. One type of such backup capacity is called operating reserve, which has to be dispatched within minutes upon request. Providing reserve capacity is lucrative because the reserve provider is rewarded even when no actual dispatch is required. Operating reserve can be provided by generating facilities that are able to quickly increase electricity supply. Alternatively, the supply-demand gap can be eliminated by reducing demand. Therefore, electricity consumers also have the opportunity to provide operating reserve if they possess the flexibility to quickly reduce their electricity consumption. Such operating reserve provided by electricity consumers is also referred to as interruptible load.

In the power systems literature, there is a large body of work on interruptible load management at the grid level. Some of the main questions addressed in 
these works are: How can interruptible load improve grid reliability and performance (Fotuhi-Firuzabad and Billinton, 2000; Bai et al., 2006; Aminifar et al., 2009)? Given offer curves from the interruptible load providers, how much interruptible load should the load serving entity procure (Tuan and Bhattacharya, 2003; Hatami et al., 2009)? For an efficient reserve market, how should interruptible load be priced (Aalami et al., 2010)? In all these models, very simplistic representations of the electricity consumers are applied. However, in order to answer the fundamental question of how much interruptible load a consumer is really able and willing to provide, a consumer's perspective has to be taken, and more detailed models have to be used.

The financial incentive for providing operating reserve reflects the value of flexible resources that can react quickly to unexpected changes in the power grid. The inherent uncertainty here is that one does not know in advance when and how much reserve will be needed. In a previous work, Zhang et al. (2015a) have considered providing operating reserve by using the power generation capability of a cryogenic energy storage system. To account for the uncertainty in reserve demand, a robust optimization approach has been applied, which also provides the flexibility of adjusting the level of conservatism in the solution. Similarly, Vujanic et al. (2012) apply robust optimization to consider the scheduling of a batch plant that provides interruptible load. Here, the uncertainty in the time of required reserve dispatch is assumed to affect the start times of the scheduled tasks. However, in the model proposed by Vujanic et al. (2012), it is assumed that the amount of interruptible load provided is known, i.e. it is not a decision variable.

In robust optimization (Ben-Tal et al., 2009), the worst case is optimized while guaranteeing feasibility for all possible realizations of the uncertainty, which is described by an uncertainty set. When providing operating reserve, dispatch upon request has to be guaranteed since otherwise, one has to pay very high penalties, or may not even be allowed to participate in the reserve market. Hence, robust optimization is a natural choice for solving problems involving operating reserve. However, a major drawback of the traditional robust optimization approach - as applied by Zhang et al. (2015a) and Vujanic et al. (2012) - is that it does not account for recourse (reactive actions after the realization of the uncertainty); hence, the solution may be overly conservative. To overcome this limitation, the concept of adjustable robust optimization has been developed in recent years (Ben-Tal et al., 2004; Kuhn et al., 2011). The main idea is to include recourse in the form of decision rules that are functions of the uncertain parameters. If tractable (typically linear) decision rules are chosen, a robust counterpart formulation can be derived in the same fashion as in traditional robust optimization. The adjustable robust optimization approach has been successfully applied to various operations research problems, such as inventory management (Ben-Tal et al., 2004), project management (Chen and Zhang, 2009), and logistics planning (Ben-Tal et al., 2011).

In this work, we develop a general mixed-integer linear programming (MILP) scheduling model for power-intensive continuous processes that participate in the reserve market by providing interruptible load. We apply an adjustable ro- 
bust optimization approach to model the uncertainty in load reduction demand under a multistage decision-making setting. A computationally tractable formulation is derived and applied to illustrative as well as real-world industrial cases.

The remainder of this paper is organized as follows. Given the problem statement in Section 2, the uncertain scheduling model is presented in Section 3. The adjustable robust counterpart is developed in Section 4. In Section 5, the proposed model is applied to an illustrative example as well as an industrial air separation case study. Finally, in Section 6, we close with a summary of the results and concluding remarks.

\section{Problem statement}

Consider a power-intensive continuously operated plant that can produce a certain set of products, for which given demands have to be satisfied. Inventory capacities exist for storable products, and additional products can be purchased at given costs. We assume that for fixed production, all production costs except for the cost of electricity are constant. In this way, for optimization purposes, the total operating cost only consists of the electricity cost and the cost of purchasing products. Electricity prices, which are time-sensitive, are assumed to be known for the scheduling horizon.

Besides selling products, the plant can gain additional revenue from providing operating reserve in the form of interruptible load, which is capacity for load reduction that the grid operator can request from the plant in case of contingency. Here, the load reduction is measured with respect to the plant's target power consumption. The interruptible load provider is rewarded regardless how much load reduction is actually required, which is uncertain. Here, we assume that besides the corresponding reduction in electricity cost, no additional payment is made to the interruptible load provider when load is actually reduced upon request. Note that depending on the operating reserve market, this assumption may be relaxed.

The goal is to find a production schedule over a given time horizon that guarantees satisfaction of all product demand under every possible realization of the uncertainty, which lies in the actual demand for load reduction. Also, the solution is considered optimal if it minimizes net operating cost for the worst case, where the net operating cost is primarily the electricity cost and product purchase cost minus the revenue from providing interruptible load. Here, we distinguish between two types of decisions: here-and-now decisions which have to be made at the beginning and cannot be changed over the course of the scheduling horizon, and wait-and-see decisions which can be adjusted after realization of the uncertainty. In this problem, the here-and-now decisions are the modes of operation, the target production rates for each product, and the committed purchase amounts for each product in each time period of the scheduling horizon. The wait-and-see decisions are the changes in production rates and product purchases if load reduction is requested or has been requested in previous time periods. 


\section{Uncertain scheduling model}

The discrete-time MILP scheduling model is based on a mode-based formulation developed in previous works (Mitra et al., 2012, 2013; Zhang et al., 2015a). Hence, we only provide brief descriptions of the scheduling constraints, and focus on the modeling of interruptible load and the derivation of the robust formulation in the next sections. Note that unless specified otherwise, all continuous variables presented in this section are constrained to be nonnegative. A list of indices, sets, parameters, and variables used in the model is given in the Nomenclature section.

\subsection{Plant model}

In this framework, we assume that the plant can operate in different operating modes, which represent operating states such as "off", "on", and "startup". The feasible region for each mode is defined by a union of convex subregions in the product space, and a linear electricity consumption function with respect to the production rates is given for each subregion. The key feature here is that every subregion has the form of a polytope. Such a model is generally referred to as a Convex Region Surrogate (CRS) model. For complex processes, CRS models can be constructed by either using a model-based (Sung and Maravelias, 2009) or a data-driven approach (Zhang et al., 2015b).

At any point in time, the plant can only run in one operating mode. For a given mode, the operating point has to lie in either one of the convex subregions. Any point in a subregion can be represented as a convex combination of the vertices of the polytope. These relationships can be expressed by the following constraints:

$$
\begin{array}{ll}
P D_{i t}=\sum_{m} \sum_{r \in R_{m}} \overline{P D}_{m r i t} & \forall i, t \in \bar{T} \\
\overline{P D}_{m r i t}=\sum_{j \in J_{m r}} \lambda_{m r j t} v_{m r j i} & \forall m, r \in R_{m}, i, t \in \bar{T} \\
\sum_{j \in J_{m r}} \lambda_{m r j t}=\bar{y}_{m r t} & \forall m, r \in R_{m}, t \in \bar{T} \\
E C_{t}=\sum_{m} \sum_{r \in R_{m}}\left(\delta_{m r} \bar{y}_{m r t}+\sum_{i} \gamma_{m r i} \overline{P D}_{m r i t}\right) & \forall t \in \bar{T} \\
y_{m t}=\sum_{r \in R_{m}} \bar{y}_{m r t} & \forall m, t \in \bar{T} \\
\sum_{m} y_{m t}=1 & \forall t \in \bar{T}
\end{array}
$$

where $R_{m}$ is the set of subregions in mode $m$, and $J_{m r}$ is the set of vertices of subregion $r \in R_{m}$. The binary variable $y_{m t}$ is 1 if mode $m$ is selected in time period $t$, whereas $\bar{y}_{m r t}$ is 1 if subregion $r \in R_{m}$ is selected in time period $t$. The amount of product $i$ produced in time period $t$ is denoted by $P D_{i t}$. Associated with $P D_{i t}$ is the disaggregated variable $\overline{P D}_{m r i t}$ for subregion $r \in R_{m}$, which is expressed as a convex combination of the corresponding vertices, $v_{m r j i}$. The 
amount of electricity consumed, $E C_{t}$, is a linear function of $P D_{i t}$ with a constant $\delta_{m r}$ and coefficients $\gamma_{m r i}$ specific to the selected subregion.

Note that an equivalent formulation is achieved if $y_{m t}$ is relaxed to be a continuous variable with $0 \leq y_{m t} \leq 1$ since according to Eq. (1e), $y_{m t}=\sum_{r \in R_{m}} \bar{y}_{m r t}$, which restricts $y_{m t}$ to be integer as $\bar{y}_{m r t}$ is binary.

\subsection{Transition constraints}

A transition occurs when the system changes from one operating point to another. In particular, constraints have to be imposed on transitions between different operating modes, which is achieved by Eqs. (2)-(4). The binary variable $z_{m m^{\prime} t}$ takes the value 1 if and only if the plant switches from mode $m$ to mode $m^{\prime}$ at time $t$, which is enforced by the following constraint:

$$
\sum_{m^{\prime} \in \overline{T R}_{m}} z_{m^{\prime} m, t-1}-\sum_{m^{\prime} \in \overline{T R}_{m}} z_{m m^{\prime}, t-1}=y_{m t}-y_{m, t-1} \quad \forall m, t \in \bar{T}
$$

where $\overline{T R}_{m}=\left\{m^{\prime}:\left(m^{\prime}, m\right) \in T R\right\}$ and $\widehat{T R}_{m}=\left\{m^{\prime}:\left(m, m^{\prime}\right) \in T R\right\}$ with $T R$ being the set of all possible mode-to-mode transitions.

The restriction that the plant has to remain in a certain mode for a minimum amount of time after a transition is stated as follows:

$$
y_{m^{\prime} t} \geq \sum_{k=1}^{\theta_{m m^{\prime}}} z_{m m^{\prime}, t-k} \quad \forall\left(m, m^{\prime}\right) \in T R, t \in \bar{T}
$$

with $\theta_{m m^{\prime}}$ being the minimum stay time in mode $m^{\prime}$ after switching to it from mode $m$.

For predefined sequences, each defined as a fixed chain of transitions from mode $m$ to mode $m^{\prime}$ to mode $m^{\prime \prime}$, we can specify a fixed stay time in mode $m^{\prime}$ by imposing the following constraint:

$$
z_{m m^{\prime}, t-\bar{\theta}_{m m^{\prime} m^{\prime \prime}}}=z_{m^{\prime} m^{\prime \prime} t} \quad \forall\left(m, m^{\prime}, m^{\prime \prime}\right) \in S Q, t \in \bar{T}
$$

where $S Q$ is the set of predefined sequences and $\bar{\theta}_{m m^{\prime} m^{\prime \prime}}$ is the fixed stay time in mode $m^{\prime}$ in the corresponding sequence.

\subsection{Mass balance constraints}

The plant produces a set of products, of which some may be storable. As stated in Eq. (5a), the inventory level for product $i$ at time $t, I V_{i t}$, is the inventory level at time $t-1$ plus the amount produced minus the amount sold, $S L_{i t}$. Eq. (5b) sets bounds on the inventory levels, and Eq. (5c) states that also products purchased from other sources, denoted by $P C_{i t}$, can be used to satisfy demand.

$$
\begin{array}{ll}
I V_{i t}=I V_{i, t-1}+P D_{i t}-S L_{i t} & \forall i, t \in \bar{T} \\
I V_{i t}^{\min } \leq I V_{i t} \leq I V_{i t}^{\max } & \forall i, t \in \bar{T} \\
S L_{i t}+P C_{i t}=D_{i t} & \forall i, t \in \bar{T}
\end{array}
$$




\subsection{Interruptible load constraints}

Interruptible load can be seen as the capability of a plant to reduce its electricity load within a short amount of time. It can hence be used as an operating reserve resource to release the stress on the power grid in times of contingency. When interruptible load is provided, the plant still operates at its planned target production level, but has to be ready to respond to load reduction requests. When such a request actually occurs, the plant has to deviate from its target production rate such that the requested load reduction is achieved.

To model the provision of interruptible load, we first replace $\overline{P D}_{\text {mrit }}$ by the following sum:

$$
\overline{P D}_{\text {mrit }}=\widehat{P D}_{m r i t}+\widetilde{P D}_{m r i t} \quad \forall m, r \in R_{m}, i, t \in \bar{T}
$$

where $\widehat{P D}_{\text {mrit }}$ is the target production rate and $\widetilde{P D}_{m r i t}$ is the response decrease in production rate when load reduction is required, in which case $\widetilde{P D}_{\text {mrit }}$ takes a negative value. The reduction in power consumption associated with the decrease in production with respect to the target production rate has to be at least the amount of requested load reduction, $L R_{t}$, as stated in the following constraint:

$$
\sum_{m} \sum_{r \in R_{m}} \sum_{i} \gamma_{m r i} \widetilde{P D}_{m r i t} \leq-L R_{t} \quad \forall t \in \bar{T}
$$

where $L R_{t}$ is an uncertain parameter whose characteristics will be discussed in detail later.

We further define a binary variable $x_{t}$, which takes the value 1 if interruptible load is provided in time period $t$. When interruptible load is provided, there may be lower and upper bounds on the provided amount as stated in the following:

$$
I L_{t}^{\min } x_{t} \leq I L_{t} \leq I L_{t}^{\max } x_{t} \quad \forall t \in \bar{T}
$$

where $I L_{t}$ is the amount of interruptible load provided in time period $t$.

\subsection{Initial conditions}

The scheduling problem is formulated for a given time horizon. For the problem to be well-defined, initial conditions are required, which are given in the following:

$$
\begin{array}{ll}
I V_{i, 0}=I V_{i}^{\mathrm{ini}} & \forall i \\
y_{m, 0}=y_{m}^{\mathrm{ini}} & \forall m \\
z_{m m^{\prime} t}=z_{m m^{\prime} t}^{\mathrm{ini}} & \forall\left(m, m^{\prime}\right) \in T R,-\theta^{u}+1 \leq t \leq-1
\end{array}
$$

with $\theta^{\max }=\max \left(\max _{m, m^{\prime}}\left\{\theta_{m m^{\prime}}\right\}, \max _{m, m^{\prime}, m^{\prime \prime}}\left\{\bar{\theta}_{m m^{\prime} m^{\prime \prime}}\right\}\right)$, which defines for how far back in the past the mode switching information has to be provided. 


\subsection{Objective function}

The objective is to minimize the total net operating cost, $T C$, which is defined as the sum of the electricity cost and the product purchase cost minus the revenue gained from providing interruptible load, as stated in the following equation:

$$
T C=\sum_{t \in \bar{T}}\left(\alpha_{t}^{\mathrm{EC}} E C_{t}+\sum_{i} \alpha_{i t}^{\mathrm{PC}} P C_{i t}-\alpha_{t}^{\mathrm{IL}} I L_{t}\right)
$$

where $\alpha_{t}^{\mathrm{EC}}, \alpha_{t}^{\mathrm{PC}}$, and $\alpha_{t}^{\mathrm{IL}}$ are price coefficients.

\subsection{Uncertain optimization problem}

The uncertainty in the model lies in the parameter $L R$ since one does not know in advance when and how much load reduction will be required. However, when a plant provides interruptible load, it has to guarantee that load reduction up to the committed amount can be achieved when it is requested; noncompliance would result in extremely high penalties, or one may not even be allowed to participate in the market. Hence, in this uncertain optimization problem, we seek a robust solution in a sense that it has to be feasible for every possible realization of the uncertain parameter.

Possible realizations of the uncertainty are defined in terms of an uncertainty set $U$. Here, the required load reduction, $L R$, cannot be higher than the amount of provided interruptible load, $I L$. Thus, the uncertainty set depends on $I L$, i.e. $U=U(I L)$; hence, this is a case of endogenous uncertainty as the uncertainty depends on the decisions made.

The uncertain optimization problem is formulated as follows:

$$
\begin{array}{ll}
\min & \max _{L R \in U(I L)}\{T C\} \\
\text { s.t. } & \text { Eqs. }(1)-(10) \quad \forall L R \in U(I L)
\end{array}
$$

where $L R=\left[L R_{1}, L R_{2}, \ldots, L R_{t^{\mathrm{fin}}}\right]^{\mathrm{T}}$ and $I L=\left[I L_{1}, I L_{2}, \ldots, I L_{t^{\mathrm{fin}}}\right]^{\mathrm{T}}$. It is stated that all constraints have to be feasible for any possible realization of $L R$ with respect to $U(I L)$. The objective is to minimize $\max _{L R \in U(I L)}\{T C\}$, which is the highest possible total net operating cost. In other words, the optimal objective function value corresponds to the worst case, which provides an upper bound on the total net operating cost for every realization of the uncertainty.

\subsection{Uncertainty set}

Given a committed amount of interruptible load in time period $t, I L_{t}, L R_{t}$ can only take values between zero and $I L_{t}$. The uncertainty set can simply be formulated as

$$
U(I L)=\left\{L R: 0 \leq L R_{t} \leq I L_{t} \forall t \in \bar{T}\right\}
$$

which is obviously very conservative since $L R_{t}$ could take the value of $I L_{t}$ for all $t$. In order to reduce the level of conservatism, we apply the "budget of 
uncertainty" approach (Bertsimas and Sim, 2004) and adopt the uncertainty set proposed by Zhang et al. (2015a):

$$
W(I L)=\left\{w:\left(L R_{t}=I L_{t} w_{t}, 0 \leq w_{k} \leq 1 \forall 1 \leq k \leq t, \sum_{k=1}^{t} w_{k} \leq \Gamma_{t}\right) \forall t \in \bar{T}\right\}
$$

where $w_{t}=L R_{t} / I L_{t}$ is the normalized required load reduction, and $\Gamma_{t}$ is a budget parameter limiting the cumulative load reduction required up to time $t$. By changing $\Gamma_{t}$, the level of conservatism can be adjusted. In practice, the budget parameters can be chosen based on historical data; alternatively, depending on the market, there may be a strict limit on the number of times in which load reduction can be requested during a specific time horizon, which can be used to set $\Gamma_{t}$. Note that in order to have the desired effect, $\Gamma_{t}$ has to be monotonically increasing with $t$.

\section{Adjustable robust counterpart}

The uncertain optimization problem given by Eqs. (11) is a semi-infinite program that cannot be readily solved. Hence, we transform it into a problem with a finite number of constraints; in particular, the new formulation guarantees feasibility for every realization of the uncertainty with respect to the uncertainty set, and is therefore referred to as the robust counterpart. Here, we apply an adjustable robust optimization approach (Ben-Tal et al., 2004) to account for recourse.

In general, wait-and-see decisions can be expressed as functions of the uncertainty; hence, we have:

$$
\begin{array}{ll}
\overline{P D}_{m r i t}=\widehat{P D}_{m r i t}+\widetilde{P D}_{m r i t}(w) & \forall m, r \in R_{m}, i, t \in \bar{T} \\
P C_{i t}=\widehat{P C}_{i t}+\widetilde{P C}_{i t}(w) & \forall i, t \in \bar{T}
\end{array}
$$

where $\widehat{P D}_{m r i t}$ and $\widehat{P C}_{i t}$ are the target production rate and the committed product purchase, respectively, which are here-and-now decisions. $\widetilde{P D}_{\text {mrit }}$ denotes the change in production rate depending on the realization of the uncertainty $w$; it includes the decrease in production rate when load reduction is requested as well as the possible increase in production rate after load reduction in order to make up for the loss in production. Similarly, $\widetilde{P C}_{i t}$ is the increase in product purchase as a function of $w$.

\subsection{Linear decision rules}

Generally, the functions of $w$ in Eqs. (14) could take any form. However, it is computationally intractable to optimize over the infinitely large set of all possible functions. Hence, in order to obtain a tractable formulation, we restrict ourselves to linear functions with respect to $w$, also referred to as linear decision rules, which are as follows:

$$
\widetilde{P D}_{m r i t}(w)=\sum_{k=t-\zeta_{t}}^{t} p_{m r i t k} w_{k} \quad \forall m, r \in R_{m}, i, t \in \bar{T}
$$




$$
\begin{array}{ll}
\widetilde{P C}_{i t}(w)=\sum_{k=t-\zeta_{t}}^{t} q_{i t k} w_{k} & \forall i, t \in \bar{T} \\
p_{\text {mritt }} \leq 0 & \forall m, r \in R_{m}, i, t \in \bar{T} \\
p_{\text {mritk }} \geq 0 & \forall m, r \in R_{m}, i, t \in \bar{T}, t-\zeta_{t} \leq k \leq t-1 \\
q_{i t k} \geq 0 & \forall i, t \in \bar{T}, t-\zeta_{t} \leq k \leq t
\end{array}
$$

where $p_{\text {mritk }}$ and $q_{i t k}$ are variables that define the linear decision rules. This formulation allows multistage decision-making since at each time period $t$, the recourse decision depends on the uncertain parameters that have been realized in the preceding $\zeta_{t}$ time periods as well as the current time period, i.e. $w_{k}$ for $t-\zeta_{t} \leq k \leq t$. The parameter $\zeta_{t}$ can be any integer between zero and $t-1$; it can be seen as another parameter for adjusting the level of conservatism. The greater $\zeta_{t}$ is, the more realized uncertainty is considered in the recourse; hence, an improved solution may be obtained. However, the problem size increases with $\zeta_{t}$. Therefore, in large-scale problems, a $\zeta_{t}<t-1$ can be chosen in order to achieve a trade-off between level of conservatism and problem size.

Eq. (15c) states that $p_{\text {mritk }}$ for $k=t$ has to be nonpositive since in case load reduction is requested in time period $t$, the plant has to react with load reduction. However, as expressed in Eq. (15d), $p_{\text {mritk }}$ is nonnegative for $k<t$ because if load was reduced in time period $k$, the natural recourse action in time period $t>k$ is an increase in production rate to make up for the loss in production. In the case of product purchase, only positive recourse makes sense; hence, $q_{i t k}$ is restricted to be nonnegative.

\subsection{Reformulation of plant model}

In order to construct the robust counterpart, we have to reformulate the CRS models that characterize the plant such that each polyhedral subregion is described by a set of inequalities instead of equalities. There are two commonly used representations of a polytope: the V-representation and the $\mathrm{H}$ representation. In the $\mathrm{V}$-representation, a point in the polytope is expressed as a convex combination of the polytope's vertices; we have applied this representation in the CRS models through Eqs. (1b)-(1c). In the H-representation, the polytope is represented by a set of linear inequalities, where each inequality corresponds to a supporting hyperplane. The V-and H-representations are illustrated in the example shown in Figure 1.

We replace Eqs. (1b)-(1c) by the following constraints which constitute the $\mathrm{H}$-representations of each subregion in the CRS models:

$$
\begin{array}{ll}
\sum_{i} a_{m r f i} \overline{P D}_{m r i t}(w) \geq b_{m r f} \bar{y}_{m r t} & \forall m, r \in R_{m}, f \in F_{m r}, t \in \bar{T} \\
0 \leq \overline{P D}_{m r i t}(w) \leq P D_{m r i}^{\max } \bar{y}_{m r t} & \forall m, r \in R_{m}, i, t \in \bar{T}
\end{array}
$$

where $a_{m r f i}$ and $b_{m r f}$ are parameters defining the inequalities, and $F_{m r}$ is the set of supporting hyperplanes associated with subregion $r$ of mode $m$. 


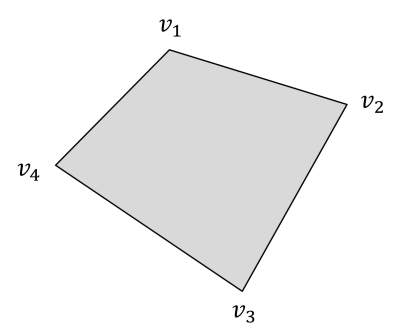

(a) V-representation

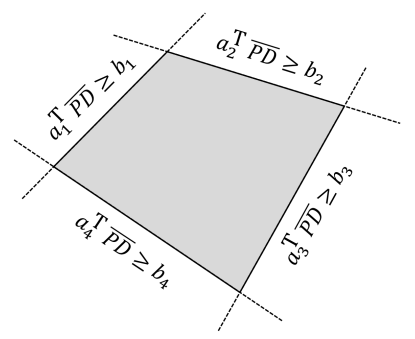

(b) H-representation

Figure 1: The V-representation makes use of the polytope's vertices; the $\mathrm{H}-$ representation is formed by the supporting hyperplanes.

\subsection{Elimination of state variables}

Now we remove all "state variables", i.e. variables that can be simply represented as linear functions of the decision variables. After eliminating the state variables, we obtain the following uncertain optimization problem:

$$
\begin{aligned}
\min \max _{w \in W(I L)}\{ & \sum_{t \in \bar{T}} \alpha_{t}^{\mathrm{EC}} \sum_{m} \sum_{r \in R_{m}}\left[\delta_{m r} \bar{y}_{m r t}+\sum_{i} \gamma_{m r i}\left(\widehat{P D}_{m r i t}+\sum_{k=t-\zeta_{t}}^{t} p_{m r i t k} w_{k}\right)\right] \\
& +\sum_{t \in \bar{T}} \sum_{i} \alpha_{i t}^{\mathrm{PC}}\left(\widehat{P C}_{i t}+\sum_{k=t-\zeta_{t}}^{t} q_{i t k} w_{k}\right)-\sum_{t \in \bar{T}} \alpha_{t}^{\mathrm{IL}} I L_{t} \\
& \left.+\sum_{m} \sum_{r \in R_{m}} \sum_{i} \sum_{t \in \bar{T}} \alpha_{m r i}^{\mathrm{RP}}\left(-p_{m r i t t}+\sum_{k=t-\zeta_{t}}^{t-1} p_{m r i t k}\right)+\sum_{i} \sum_{t \in \bar{T}} \sum_{k=t-\zeta_{t}}^{t} \alpha_{i}^{\mathrm{RQ}} q_{i t k}\right\}
\end{aligned}
$$

s.t. $\quad$ Eqs. (1e)-(1f), (2)-(4), (8), (9b)-(9c), (15c)-(15e)

$$
\begin{aligned}
& \sum_{i} a_{m r f i}\left(\widehat{P D}_{m r i t}+\sum_{k=t-\zeta_{t}}^{t} p_{m r i t k} w_{k}\right) \geq b_{m r f} \bar{y}_{m r t} \quad \forall m, r \in R_{m}, f \in F_{m r}, t \in \bar{T} \\
& 0 \leq \widehat{P D}_{m r i t}+\sum_{k=t-\zeta_{t}}^{t} p_{m r i t k} w_{k} \leq P D_{m r i}^{\max } \bar{y}_{m r t} \quad \forall m, r \in R_{m}, i, t \in \bar{T} \quad(17 \mathrm{c}) \\
& I V_{i}^{\min } \leq I V_{i, 0}+\sum_{k=1}^{t}\left[\sum_{m} \sum_{r \in R_{m}}\left(\widehat{P D}_{m r i k}+\sum_{l=k-\zeta_{k}}^{k} p_{m r i k l} w_{l}\right)+\left(\widehat{P C}_{i k}+\sum_{l=k-\zeta_{k}}^{k} q_{i k l} w_{l}\right)-D_{i k}\right] \\
& \leq I V_{i}^{\max } \quad \forall i, t \in \bar{T} \\
& \sum_{m} \sum_{r \in R_{m}} \sum_{i} \gamma_{m r i} \sum_{k=t-\zeta_{t}}^{t} p_{m r i t k} w_{k} \leq-I L_{t} w_{t}+\Omega\left(1-x_{t}\right) \quad \forall t \in \bar{T} \\
& \widehat{P D}_{m r i t} \geq 0 \quad \forall m, r \in R_{m}, i, t \in \bar{T} \\
& \widehat{P C}_{i t} \geq 0 \quad \forall i, t \in \bar{T}
\end{aligned}
$$




$$
\begin{aligned}
& x_{t} \in\{0,1\} \quad \forall t \in \bar{T} \\
& y_{m t} \in\{0,1\} \quad \forall m, t \in \bar{T} \\
& \bar{y}_{m r t} \in\{0,1\} \quad \forall m, r \in R_{m}, t \in \bar{T} \\
& z_{m m^{\prime} t} \in\{0,1\} \quad \forall\left(m, m^{\prime}\right) \in T R, t \in \bar{T} \\
& \forall w \in W(I L)
\end{aligned}
$$

where costs for recourse actions have been added to the objective function. $\Omega$ is a big-M parameter, which allows positive recourse in production. The last line states that all equations have to simultaneously hold for all $w \in W(I L)$.

Note that the smallest possible value for $\Omega$ is $\max _{m, r \in R_{m}} \Delta E C_{m r}^{\max }$ with

$$
\begin{aligned}
& \Delta E C_{m r}^{\max }=\max \sum_{i} \gamma_{m r i}\left(P D_{m r i}^{\mathrm{up}}-P D_{m r i}^{\mathrm{lo}}\right) \\
& \text { s.t. } \sum_{i} a_{m r f i} P D_{m r i}^{\text {up }} \geq b_{m r f} \quad \forall f \in F_{m r} \\
& \sum_{i} a_{m r f i} P D_{m r i}^{\mathrm{lo}} \geq b_{m r f} \quad \forall f \in F_{m r}
\end{aligned}
$$

where $\Delta E C_{m r}^{\max }$ is the maximum load change that can be achieved in subregion $r$ of mode $m$.

\subsection{Linearly adjustable robust counterpart}

By using techniques commonly applied in robust optimization, the uncertain optimization problem with recourse given by Eqs. (17) can be reformulated into a finite-dimensional problem. The resulting linearly adjustable robust counterpart (ARC), for which the detailed derivation is presented in Appendix A, is shown in the following:

$$
\begin{aligned}
\min \sum_{t \in \bar{T}}[ & {\left[\sum_{m} \sum_{r \in R_{m}} \alpha_{t}^{\mathrm{EC}}\left(\delta_{m r} \bar{y}_{m r t}+\sum_{i} \gamma_{m r i} \widehat{P D}_{m r i t}\right)+\sum_{i} \alpha_{i t}^{\mathrm{PC}} \widehat{P C}_{i t}-\alpha_{t}^{\mathrm{IL}} I L_{t}\right.} \\
& \left.+\sum_{m} \sum_{r \in R_{m}} \sum_{i} \alpha_{m r i}^{\mathrm{RP}}\left(-p_{m r i t t}+\sum_{k=t-\zeta_{t}}^{t-1} p_{m r i t k}\right)+\sum_{i} \sum_{k=t-\zeta_{t}}^{t} \alpha_{i}^{\mathrm{RQ}} q_{i t k}\right]+\left(\Gamma_{t^{\mathrm{fin}}} u^{\mathrm{A}}+\sum_{k=1}^{t^{\mathrm{fin}}} s_{k}^{\mathrm{A}}\right)
\end{aligned}
$$

s.t. $\quad$ Eqs. (1e)-(1f), (2)-(4), (8), (9b)-(9c), (15c)-(15e), (17f)-(17k)

$$
\begin{aligned}
& \sum_{i} a_{m r f i} \widehat{P D}_{m r i t}+\left(\Gamma_{t} u_{m r f t}^{\mathrm{B}}+\sum_{k=t-\zeta_{t}}^{t} s_{m r f t k}^{\mathrm{B}}\right) \geq b_{m r f} \bar{y}_{m r t} \quad \forall m, r \in R_{m}, f \in F_{m r}, t \in \bar{T} \\
& \widehat{P D}_{m r i t}+\left(\Gamma_{t} u_{m r i t}^{\mathrm{C}}+\sum_{k=t-\zeta_{t}}^{t} s_{m r i t k}^{\mathrm{C}}\right) \leq P D_{m r i}^{\mathrm{max}} \bar{y}_{m r t} \quad \forall m, r \in R_{m}, i, t \in \bar{T} \\
& \widehat{P D}_{m r i t}+\left(\Gamma_{t} u_{m r i t}^{\mathrm{D}}+\sum_{k=t-\zeta_{t}}^{t} s_{m r i t k}^{\mathrm{D}}\right) \geq 0 \quad \forall m, r \in R_{m}, i, t \in \bar{T}
\end{aligned}
$$




$$
\begin{aligned}
& I V_{i, 0}+\sum_{k=1}^{t}\left(\sum_{m} \sum_{r \in R_{m}} \widehat{P D}_{m r i k}+\widehat{P C}_{i k}-D_{i k}\right)+\left(\Gamma_{t} u_{i t}^{\mathrm{E}}+\sum_{k=1}^{t} s_{i t k}^{\mathrm{E}}\right) \leq I V_{i}^{\mathrm{max}} \quad \forall i, t \in \bar{T} \\
& I V_{i, 0}+\sum_{k=1}^{t}\left(\sum_{m} \sum_{r \in R_{m}} \widehat{P D}_{m r i k}+\widehat{P C}_{i k}-D_{i k}\right)+\left(\Gamma_{t} u_{i t}^{\mathrm{F}}+\sum_{k=1}^{t} s_{i t k}^{\mathrm{F}}\right) \geq I V_{i}^{\mathrm{min}} \quad \forall i, t \in \bar{T} \\
& \Gamma_{t} u_{t}^{\mathrm{G}}+\sum_{k=t-\zeta_{t}}^{t} s_{t k}^{\mathrm{G}} \leq \Omega\left(1-x_{t}\right) \quad \forall t \in \bar{T} \\
& u^{\mathrm{A}}+s_{k}^{\mathrm{A}} \geq \sum_{l=k}^{k+\zeta_{k}} \sum_{i}\left(\sum_{m} \sum_{r \in R_{m}} \alpha_{l}^{\mathrm{EC}} \gamma_{m r i} p_{m r i l k}+\alpha_{i l}^{\mathrm{PC}} q_{i l k}\right) \quad \forall 1 \leq k \leq t^{\mathrm{fin}} \\
& u^{\mathrm{A}} \geq 0 \quad \forall \\
& s_{k}^{\mathrm{A}} \geq 0 \quad \forall 1 \leq k \leq t^{\mathrm{fin}} \\
& u_{m r f t}^{\mathrm{B}}+s_{m r f t k}^{\mathrm{B}} \leq \sum_{i} a_{m r f i} p_{m r i t k} \quad \forall m, r \in R_{m}, f \in F_{m r}, t \in \bar{T}, t-\zeta_{t} \leq k \leq t \\
& u_{m r f t}^{\mathrm{B}} \leq 0 \quad \forall m, r \in R_{m}, f \in F_{m r}, t \in \bar{T} \\
& s_{m r f t k}^{\mathrm{B}} \leq 0 \quad \forall m, r \in R_{m}, f \in F_{m r}, t \in \bar{T}, t-\zeta_{t} \leq k \leq t \\
& u_{m r i t}^{\mathrm{C}}+s_{m r i t k}^{\mathrm{C}} \geq p_{m r i t k} \quad \forall m, r \in R_{m}, i, t \in \bar{T}, t-\zeta_{t} \leq k \leq t \\
& u_{m r i t}^{\mathrm{C}} \geq 0 \quad \forall m, r \in R_{m}, i, t \in \bar{T} \\
& s_{m r i t k}^{\mathrm{C}} \geq 0 \quad \forall m, r \in R_{m}, i, t \in \bar{T}, t-\zeta_{t} \leq k \leq t \\
& u_{m r i t}^{\mathrm{D}}+s_{m r i t k}^{\mathrm{D}} \leq p_{m r i t k} \quad \forall m, r \in R_{m}, i, t \in \bar{T}, t-\zeta_{t} \leq k \leq t \\
& u_{m r i t}^{\mathrm{D}} \leq 0 \quad \forall m, r \in R_{m}, i, t \in \bar{T} \\
& s_{m r i t k}^{\mathrm{D}} \leq 0 \quad \forall m, r \in R_{m}, i, t \in \bar{T}, t-\zeta_{t} \leq k \leq t \\
& u_{i t}^{\mathrm{E}}+s_{i t k}^{\mathrm{E}} \geq \sum_{l=k}^{k+\zeta_{k}}\left(\sum_{m} \sum_{r \in R_{m}} p_{m r i l k}+q_{i l k}\right) \quad \forall i, t \in \bar{T}, 1 \leq k \leq t \\
& u_{i t}^{\mathrm{E}} \geq 0 \quad \forall i, t \in \bar{T} \\
& s_{i t k}^{\mathrm{E}} \geq 0 \quad \forall i, t \in \bar{T}, 1 \leq k \leq t \\
& u_{i t}^{\mathrm{F}}+s_{i t k}^{\mathrm{F}} \leq \sum_{l=k}^{k+\zeta_{k}}\left(\sum_{m} \sum_{r \in R_{m}} p_{m r i l k}+q_{i l k}\right) \quad \forall i, t \in \bar{T}, 1 \leq k \leq t \\
& u_{i t}^{\mathrm{F}} \leq 0 \quad \forall i, t \in \bar{T} \\
& s_{i t k}^{\mathrm{F}} \leq 0 \quad \forall i, t \in \bar{T}, 1 \leq k \leq t \\
& u_{t}^{\mathrm{G}}+s_{t k}^{\mathrm{G}} \geq \sum_{m} \sum_{r \in R_{m}} \sum_{i} \gamma_{m r i} p_{m r i t k} \quad \forall t \in \bar{T}, t-\zeta_{t} \leq k \leq t-1
\end{aligned}
$$




$$
\begin{aligned}
& u_{t}^{\mathrm{G}}+s_{t t}^{\mathrm{G}} \geq \sum_{m} \sum_{r \in R_{m}} \sum_{i} \gamma_{m r i} p_{\text {mritt }}+I L_{t} \quad \forall t \in \bar{T} \\
& u_{t}^{\mathrm{G}} \geq 0 \quad \forall t \in \bar{T} \\
& s_{t k}^{\mathrm{G}} \geq 0 \quad \forall t \in \bar{T}, t-\zeta_{t} \leq k \leq t
\end{aligned}
$$

\subsection{Remark on robust formulation without recourse}

Usually, the adjustable robust optimization approach is compared to the traditional robust optimization approach, which does not consider recourse. However, it turns out that formulating a robust counterpart without recourse for this problem is not generally possible without making further restrictive assumptions.

In order to account for uncertainty without the notion of recourse, the effect of the uncertainty on the model has to be exactly known. Typically, for tractability reasons, one has to be able to express this effect as a linear function of the uncertain parameters, which in our case are the load reduction demands in each time period. The necessary response to a load reduction request is a corresponding decrease in production, which is expressed in Eq. (7). However, this response is not unique since with multiple products, there may be an infinite number of possible combinations of production rates that lead to the same amount of load reduction. Therefore, a robust model without recourse cannot be formulated unless extremely conservative assumptions are made, e.g. by fixing the production rates for all but one products a priori. For the limiting case of one single product, however, a traditional robust optimization model without recourse can be formulated. To keep the paper concise, the derivation of the corresponding robust counterpart is shown in the supplementary material.

\subsection{Tightening constraints}

In order to further improve the computational performance of the MILP model, redundant constraints are added that lead to a tighter formulation. First, we notice that the set of operating modes, $M$, can be divided into two disjoint sets: a set of flexible modes and a set of inflexible modes, denoted by $\bar{M}$ and $\widehat{M}$, respectively. In a flexible mode, the electricity consumption can be changed within a range; hence, it is suited for providing interruptible load. In contrast, an inflexible mode is represented by one single operating point and does not possess the flexibility for load adjustment. Inflexible modes are, for example, the off mode and modes representing transitional stages, such as startup and shutdown. With this insight, we know that all $p_{\text {mritk }}$ associated with inflexible modes have to be zero. Therefore, we can remove these $p$-variables as well as all corresponding constraints that result from the duals of the lower-level problems. By doing so, the numbers of variables and constraints can potentially be significantly reduced.

The same insight can be used to formulate the following tightening constraints:

$$
x_{t} \leq \sum_{m \in \bar{M}} y_{m t} \quad \forall t \in \bar{T}
$$




$$
x_{t} \leq 1-y_{m t} \quad \forall m \in \widehat{M}, t \in \bar{T}
$$

where Eq. (20a) states that $x_{t}$ can only take the value 1 if a flexible mode is chosen in time period $t$. Similarly, Eq. (20b) states that $x_{t}$ has to take the value 0 if an inflexible mode is selected in time period $t$.

\section{Case studies}

In the following, the proposed robust model is applied to an illustrative example as well as a real-world industrial air separation case. All models were implemented in GAMS 24.4.1 (GAMS Development Corporation, 2015), and the commercial solver CPLEX 12.6.1 was applied to solve the MILPs on an Intel ${ }^{\circledR}$ Core $^{\mathrm{TM}}$ i7-2600 machine at $3.40 \mathrm{GHz}$ with 8 processors and 8 GB RAM running Windows 7 Professional.

\subsection{Illustrative example}

In order to demonstrate the features of the model, we first apply it to a small illustrative example in which a single-product plant is considered. The scheduling problem is solved for a time horizon of $48 \mathrm{~h}$ while applying an hourly time discretization.

The given plant can operate in three different operating modes: off, startup, and on, where each mode is represented by one single convex region. Table 1 shows the constraints for the H-representations of each mode as well as the corresponding fixed and unit electricity consumption parameters. Note that only the on mode has the flexibility for load changes. Table 2 shows the possible mode transitions and the respective minimum stay times. Furthermore, in the predefined sequence off $\rightarrow$ startup $\rightarrow$ on, the plant has to remain in the startup mode for exactly $4 \mathrm{~h}$.

Table 1: H-representations, fixed $\left(\delta_{m}\right)$ and unit $\left(\gamma_{m}\right)$ electricity consumption for each operating mode (product and subregion indices have been omitted).

\begin{tabular}{cccc}
\hline Operating Mode & H-Representation & $\boldsymbol{\delta}_{\boldsymbol{m}}[\mathrm{kWh}]$ & $\gamma_{\boldsymbol{m}}[\mathrm{kWh} / \mathbf{k g}]$ \\
\hline off & $0 \leq P D_{m t} \leq 0$ & 0 & 0 \\
startup & $5 \leq P D_{m t} \leq 5$ & 0 & 60 \\
on & $80 \leq P D_{m t} \leq 160$ & 1500 & 20 \\
\hline
\end{tabular}


Table 2: Possible transitions between the different operating modes and the corresponding minimum stay times.

\begin{tabular}{cc}
\hline $\begin{array}{c}\text { Transition from } \\
\text { Mode } \boldsymbol{m} \text { to Mode } \boldsymbol{m}^{\prime}\end{array}$ & $\begin{array}{c}\text { Minimum Stay } \\
\text { Time in Mode } \boldsymbol{m}^{\prime}[\mathbf{h}]\end{array}$ \\
\hline off $\rightarrow$ startup & 4 \\
startup $\rightarrow$ on & 6 \\
on $\rightarrow$ off & 8 \\
\hline
\end{tabular}

At the beginning of the scheduling horizon, the plant is operating in the on mode, and it is assumed that no mode switching has occurred in the eight time periods before the beginning of the scheduling horizon. The initial inventory is $1,000 \mathrm{~kg}$. The minimum and maximum inventory levels are set to 0 and $5,000 \mathrm{~kg}$, respectively, for all time points. The only exception is the end of the scheduling horizon at which the amount of product in inventory is constrained to be at least $1,000 \mathrm{~kg}$.

When interruptible load is provided in a time period, the provided amount has to be between 200 and $1,600 \mathrm{kWh}$. The electricity and interruptible load prices are shown in Figure 2. The price for purchasing additional products is $\$ 3 / \mathrm{kg}$, and the unit costs for recourse, $\alpha^{\mathrm{RP}}$ and $\alpha^{\mathrm{RQ}}$, are set to $\$ 0.01 / \mathrm{kg}$ for all time periods.

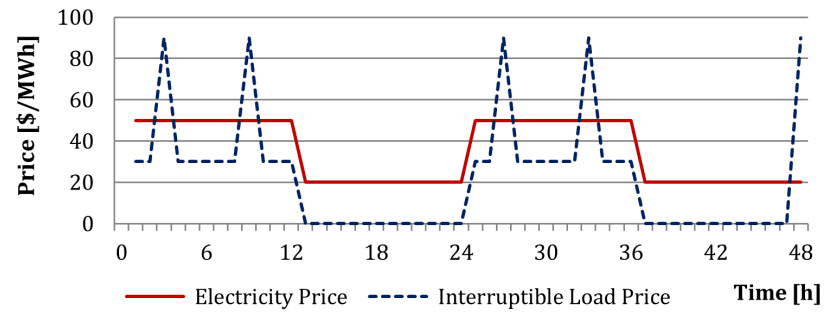

Figure 2: Electricity and interruptible load prices for the illustrative example.

Figure 3 shows the results of the base case, in which the provision of interruptible load is not considered. Note that in the figure, the y-axes for the inventory profile and the product flows are shown on the left and right hand side, respectively. Positive columns indicate accumulation of product, while negative columns (demand) indicate depletion of product. The level of plant utilization, which is defined as the ratio between the amount of product produced and the maximum amount that can be produced, is at approximately $77 \%$, with the plant's maximum production capacity being $160 \mathrm{~kg} / \mathrm{h}$. One can see that the plant is shut down for several hours $(24-33 \mathrm{~h})$ while still satisfying product demand. A small amount of product has to be purchased, yet the cost is greatly outweighed by the savings in electricity cost made possible by the temporary shutdown of the plant. The inventory level reaches its allowed minimum at the end of the scheduling horizon. The total cost for this optimal solution is $\$ 5,556$. 


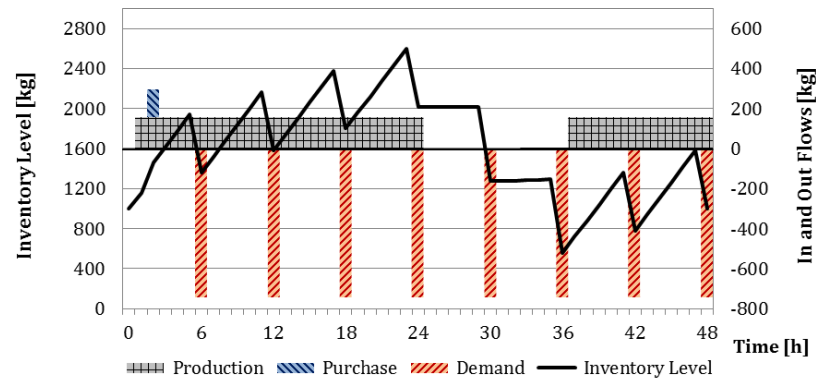

Figure 3: Product flows and resulting inventory profile for the case without interruptible load.

In the following, the effect of interruptible load is evaluated for three types of scenarios, each varying one model parameter:

1. The possible extent of recourse decisions is varied by adjusting $\zeta_{t}$. We introduce an auxiliary parameter $\bar{\zeta}$, which denotes the maximum number of previously realized uncertain parameters that are considered in the decision rules, and set $\zeta_{t}$ such that $\zeta_{t}=\min \{\bar{\zeta}, t-1\}$. The level of conservatism decreases, but the model size increases with $\bar{\zeta}$.

2. The level of uncertainty depends on the definition of the uncertainty set. It is varied by adjusting the budget parameters $\Gamma_{t}$.

3. The level of plant utilization is varied by changing the demand.

\subsubsection{Scenario type 1: Varying extent of recourse}

In order to show the value of recourse, different instances are created by varying $\bar{\zeta}$. The flexibility in the recourse actions increases with $\bar{\zeta}$ since more uncertain parameters from preceding time periods are taken into account and more terms appear in the linear decision rules. For all cases of scenario type 1 , the level of uncertainty is the same; here, the budget parameter $\Gamma_{t}$ increases every 8 time periods by 1 , i.e. maximum load reduction can only be requested once during the first $8 \mathrm{~h}$, twice during the first $16 \mathrm{~h}$, three times during the first $24 \mathrm{~h}$, etc., and at most six times during all $48 \mathrm{~h}$.

For the case of $\bar{\zeta}=0$, i.e. the recourse decision in time period $t$ only depends on the uncertainty revealed in the same time period, the results are shown in Figures 4 and 5. Along with the electricity and interruptible load prices, Figure 4 shows the target load profile for the plant as well as the amount of interruptible load provided, which obviously has to be less than the target electricity consumption. Unlike in the case without interruptible load (c.f. Figure 3), the plant is not shut down in the middle but towards the end of the scheduling horizon, allowing the provision of large amount of interruptible load when the price is high.

Figure 5 shows the inventory profile and the corresponding product flows for the case of $\bar{\zeta}=0$. In addition to the target production and purchase, the recourse 


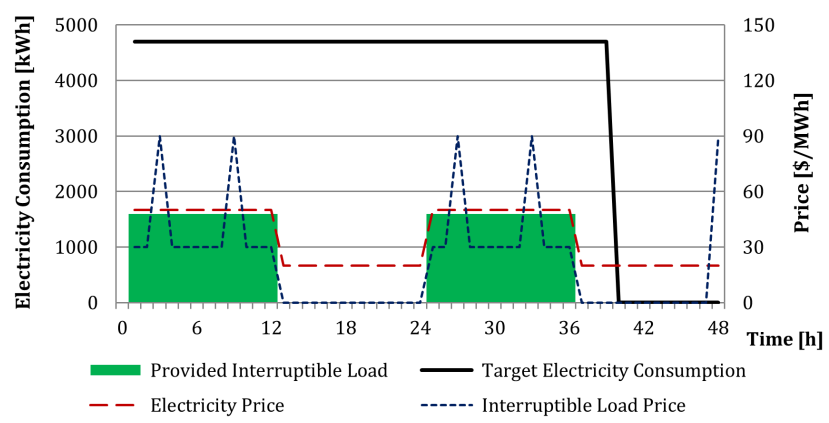

Figure 4: Target electricity consumption profile and provided interruptible load for the case of $\bar{\zeta}=0$, and price profiles.

actions in terms of reducing production (negative) and increasing purchase (positive) are shown. Negative production recourse indicates time periods in which interruptible load is provided. Since $\bar{\zeta}=0$, when load reduction is requested, the only way to regain lost production is through additional product purchase. However, since purchasing products is expensive, not all lost production is made up in the optimal solution. Instead, the solution suggests to overproduce such that an inventory buffer is created, which guarantees that all demand can be satisfied in every possible load reduction scenario. This inventory buffer is indicated by the final inventory level that is higher than its allowed minimum of $1,000 \mathrm{~kg}$. The resulting worst-case total cost is $\$ 5,540$, which is an almost negligible cost reduction of $0.3 \%$ compared to the case without interruptible load.

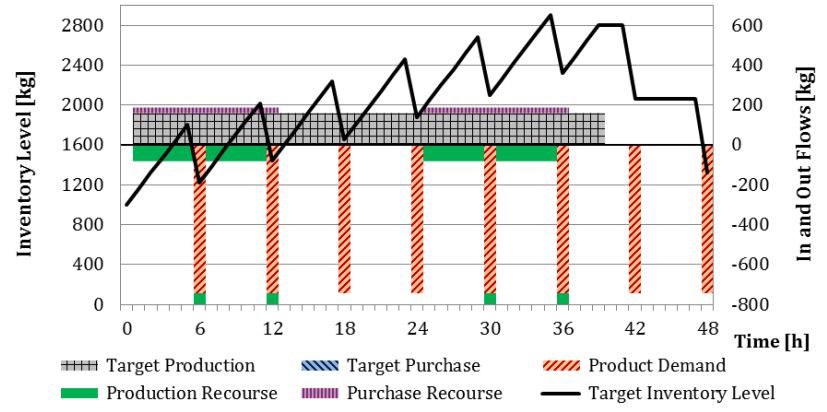

Figure 5: Target and recourse product flows and target inventory profile for the case of $\bar{\zeta}=0$.

To improve the solution while robustifying against the same level of uncertainty, $\bar{\zeta}$ is set to 47 , which is the case with the highest possible recourse flexibility since at each time period, all previous uncertain parameters in the scheduling horizon are considered in the decision rule. Hence, recourse in the 
form of production increase can also be considered. Figure 6 shows the optimal solution for this case. Here, cumulative recourse actions are shown, i.e. for each time period $t$, we plot $\sum_{k=t-\zeta_{t}}^{t} p_{t k}$ and $\sum_{k=t-\zeta_{t}}^{t} q_{t k}$. Several differences to the solution for the case of $\bar{\zeta}=0$ can be observed:

- The plant is shut down during some high-price hours in the middle (24$33 \mathrm{~h}$ ) rather than towards the end of the scheduling horizon.

- Less interruptible load is provided.

- Most lost production is made up by increasing production after the requested load reduction has occurred.

- No inventory buffer is created.

The total cost for this case is $\$ 5,127$, which is a cost reduction of $7.7 \%$ compared to the case without interruptible load. The cost savings are increased by more than 25 times compared to the case of $\bar{\zeta}=0$, simply achieved by a more flexible and realistic modeling of the recourse.

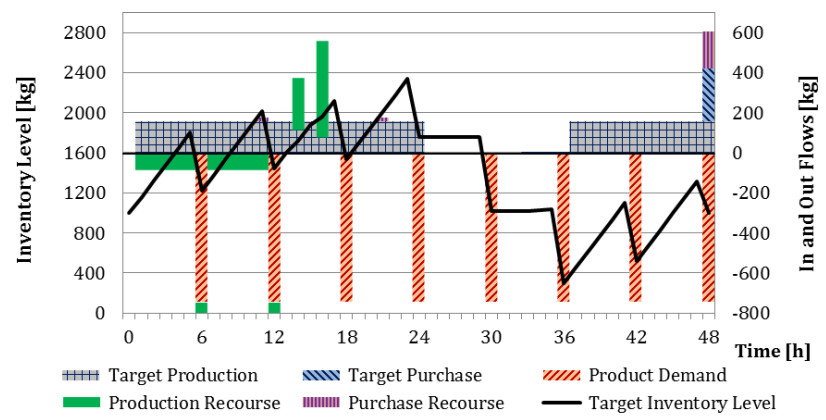

Figure 6: Target and recourse product flows and target inventory profile for the case of $\bar{\zeta}=47$.

Table 3 lists the cost values for various cases with different $\bar{\zeta}$. Note that the uncertainty-related worst-case cost- expressed in the term $\Gamma_{t^{\mathrm{fin}}} u^{\mathrm{A}}+\sum_{k=1}^{t^{\mathrm{fin}}} s_{k}^{\mathrm{A}}$ of the objective function - is not listed in the table because it is zero in all cases. One can see that the total cost decreases with $\bar{\zeta}$. Another observation is that the results for $\bar{\zeta}=23$ and $\bar{\zeta}=47$ are identical, which indicates that one may not require the model allowing maximum flexibility in the recourse to achieve the optimal solution. This is a useful insight since the model size increases with $\bar{\zeta}$; hence, there is a trade-off between level of conservatism and computational tractability. In this small example problem, the impact of increased model size is insignificant. However, as we will show in the industrial case study in Section 5.2 , it can cause severe deterioration of the computational performance. 
Table 3: Costs and revenues in $\$$ for cases with different $\bar{\zeta}$. Here, $C^{\mathrm{PD}}, C^{\mathrm{PC}}$, $R^{\mathrm{IL}}, C^{\mathrm{RC}}$, and $T C$ denote production cost, purchasing cost, revenue from providing interruptible load, recourse cost, and total cost, respectively.

\begin{tabular}{cccccc}
\hline $\bar{\zeta}$ & $\boldsymbol{C}^{\mathrm{PD}}$ & $\boldsymbol{C}^{\mathrm{PC}}$ & $\boldsymbol{R}^{\mathrm{IL}}$ & $\boldsymbol{C}^{\mathrm{RC}}$ & $\boldsymbol{T C}$ \\
\hline 0 & 7,050 & 0 & 1,536 & 26 & 5,540 \\
5 & 5,024 & 900 & 554 & 13 & 5,383 \\
11 & 5,087 & 789 & 703 & 17 & 5,190 \\
23 & 5,087 & 789 & 768 & 19 & 5,127 \\
47 & 5,087 & 789 & 768 & 19 & 5,127 \\
\hline
\end{tabular}

\subsubsection{Scenario type 2: Varying level of uncertainty}

Having examined cases with moderate uncertainty, we now vary the level of uncertainty and in particular investigate the two extremes: the most uncertain case in which load reduction can be requested in all time periods $(\Gamma=$ $\left.[1,2, \ldots, 48]^{\mathrm{T}}\right)$, and the least uncertain case in which load reduction request can only occur in one time period $\left(\Gamma=[1,1, \ldots, 1]^{\mathrm{T}}\right)$. In both cases, $\bar{\zeta}$ is chosen to be 47 .

As expected, the cost savings decrease with increasing level of uncertainty. In the most uncertain case, the total cost amounts to $\$ 5,525$, while it is only $\$ 4,851$ in the least uncertain case. It is worth taking a closer look at the results for the case with the highest level of uncertainty shown in Figure 7. Similar to the $\bar{\zeta}=0$ case from Section 5.1.1 (c.f. Figure 5), the plant is only shut down towards the end of the scheduling horizon. However, significantly less interruptible load is provided. No inventory buffer is created; hence, since the solution has to be feasible for the particular scenario in which maximum load reduction is requested in all time periods, the total amount of regained production through recourse is the same as the total amount of interruptible load provided (in terms of decrease in production).

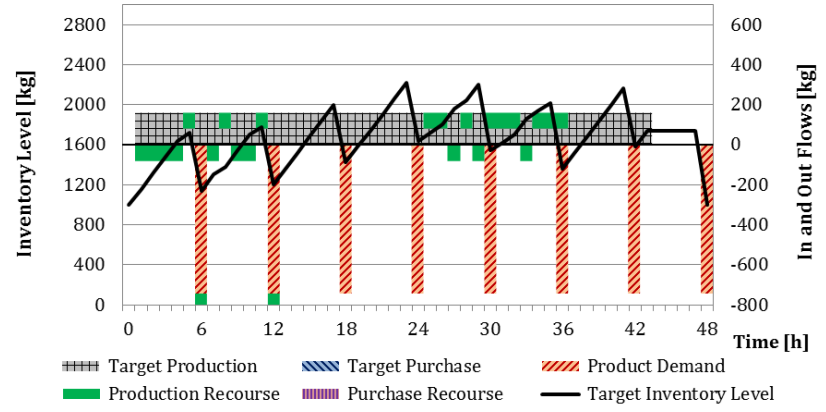

Figure 7: Target and recourse product flows and target inventory profile for the case of $\bar{\zeta}=47$ and $\Gamma=[1,2, \ldots, 48]^{\mathrm{T}}$. 


\subsubsection{Scenario type 3: Varying plant utilization}

One main insight drawn from existing works on scheduling of power-intensive industrial processes is that the benefits of DR typically decrease with the level of plant utilization (Mitra et al., 2012; Castro et al., 2013; Zhang et al., 2015a). The explanation is that lower utilization implies higher process flexibility, which allows more effective load shifting. However, when interruptible load is provided, the relationship between plant utilization and cost savings is not that obvious. On the one hand, it is still true that flexibility decreases with plant utlization; on the other hand, higher utilization implies higher target production levels, which allow more interruptible load to be provided.

We apply the uncertainty set stated in Section 5.1 .1 , set $\bar{\zeta}=47$, and solve the problem for different levels of utilization. In the diagram shown in Figure 8 , the absolute cost savings are plotted against the level of plant utilization. Before very high levels of utilization are reached, the general trend is that cost savings increase with plant utilization. However, the benefits decrease after a certain point because if the plant has to operate at almost full capacity to satisfy demand, recourse has to increasingly rely on additional product purchase, which is relatively expensive. In this case, the highest cost savings are achieved at a plant utilization of $95 \%$.

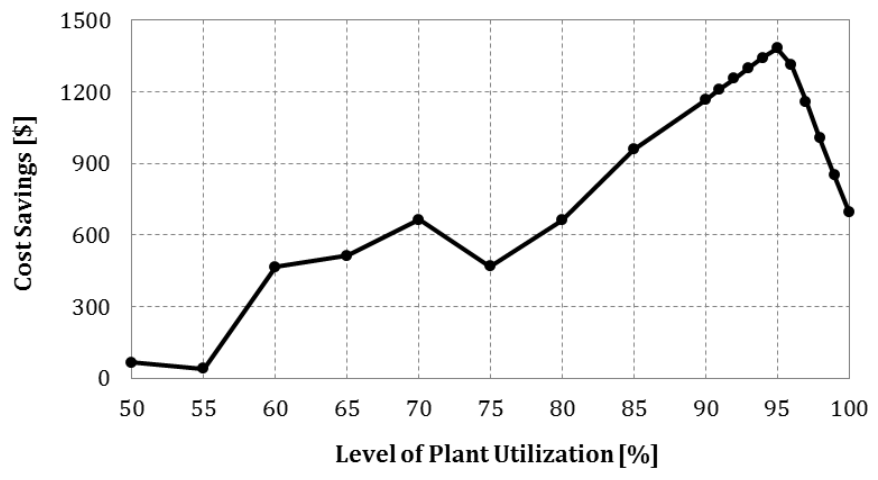

Figure 8: Absolute cost savings vs. level of plant utilization in the illustrative example.

\subsection{Industrial case study}

We now apply the proposed model to a real-world industrial case study provided by Praxair. Here, we consider an air separation plant that produces liquid oxygen (LO2) and liquid nitrogen (LN2). The scheduling horizon is one week, to which an hourly time discretization is applied resulting in 168 time periods. Note that due to confidentiality reasons, we cannot disclose information about the plant specifications as well as the actual product demand. Therefore, all results presented in the following are given without units and the values are normalized if necessary. The PJM electricity market is considered, and the 
hourly day-ahead energy and operating reserve prices are taken from the week of June 23 to 29, 2014 (PJM Interconnection LLC, 2013b,a).

A base case is considered in which plant utilization is at $90 \%$. For all instances, $\Gamma_{t}$ is chosen to increase by 1 every 24 time periods, i.e. load reduction can be requested up to 7 times over the whole week. This is a fairly conservative assumption since typically, dispatch of operating reserve is only requested a few times in months (EnerNOC, 2014). Figure 9 shows the LO2 and LN2 production, purchase, and inventory profiles for the case in which no interruptible load is provided. The optimal solution suggests to shift production as much as possible to times when the electricity price (shown in Figure 10) is low. The resulting total cost is 100 (normalized).
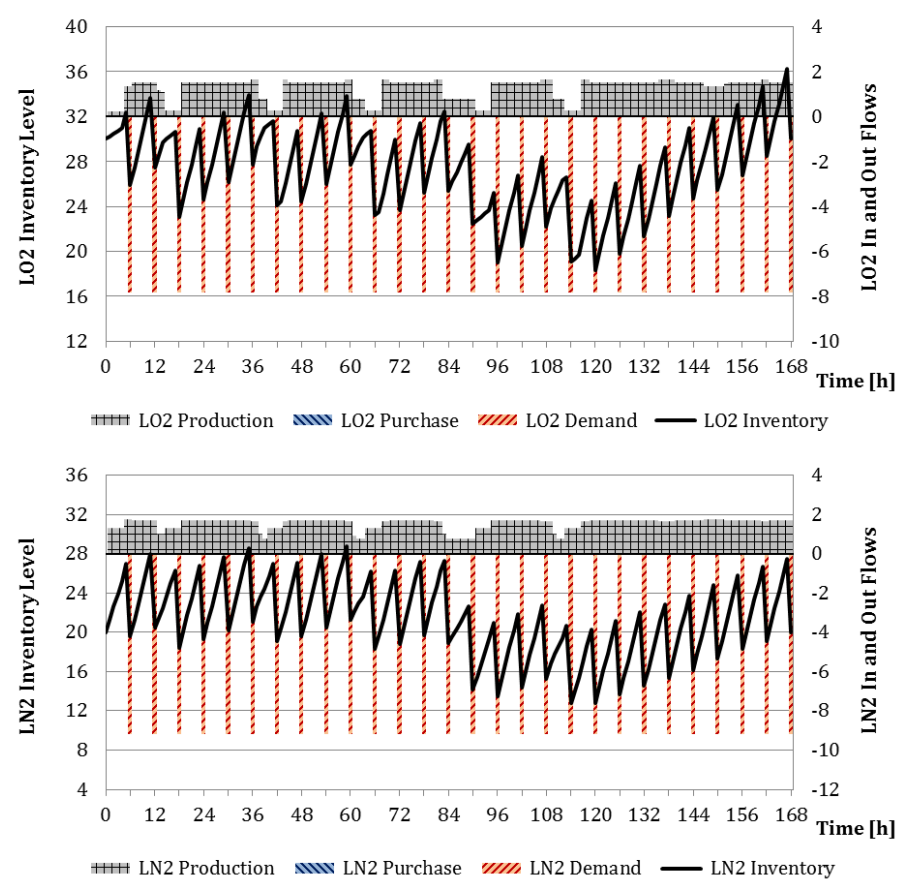

Figure 9: Product flows and inventory profiles for the case without interruptible load.

For the case when interruptible load is provided, various instances with different $\bar{\zeta}$ are created. For each instance, Table 4 lists the total costs, model sizes, and wall-clock computation times used to solve the MILPs to $0.1 \%$ optimality gap. As expected, the total cost decreases with increasing $\bar{\zeta}$ due to the higher flexibility in the recourse. However, the numbers of continuous variables and constraints grow with $\bar{\zeta}$, and lead to dramatic increases in computation time. Here, we see the clear trade-off between the level of conservatism in the model and its computational performance. For our further analysis, we choose $\bar{\zeta}$ to be 23 , as the required computation time is reasonable for practical purposes and 
only minor improvement is achieved for $\bar{\zeta}>23$.

Table 4: Total costs, model sizes, and computation times for cases with different $\bar{\zeta}$.

\begin{tabular}{cccccc}
\hline$\overline{\boldsymbol{\zeta}}$ & $\boldsymbol{T C}$ & $\begin{array}{c}\text { \# of bin. } \\
\text { variables }\end{array}$ & $\begin{array}{c}\text { \# of cont. } \\
\text { variables }\end{array}$ & $\begin{array}{c}\text { \# of } \\
\text { constraints }\end{array}$ & $\begin{array}{c}\text { Wall-Clock } \\
\text { Time [s] }\end{array}$ \\
\hline 0 & 98.83 & 3,282 & 82,670 & 84,604 & 185 \\
5 & 98.48 & 3,282 & 139,595 & 139,879 & 1,056 \\
11 & 98.33 & 3,282 & 205,628 & 203,998 & 2,598 \\
17 & 98.29 & 3,282 & 269,177 & 265,705 & 5,846 \\
23 & 98.20 & 3,282 & 330,242 & 325,000 & 6,476 \\
35 & 98.16 & 3,282 & 444,920 & 436,354 & 18,381 \\
47 & 98.14 & 3,282 & 549,662 & 538,060 & 23,280 \\
\hline
\end{tabular}

Besides the electricity and interruptible load prices, Figure 10 shows the target load profile and the amount of interruptible load provided for the case of $\bar{\zeta}=23$. It is worth pointing out that typically, as it is also the case here, high electricity prices coincide with high interruptible load prices. For those time periods, the optimal solution reveals whether reducing production to save electricity cost or increasing production to provide more interruptible load is more beneficial. Figure 11 shows the target production, purchase, and inventory profiles as well as the recourse actions, which suggest to make up for the vast majority of the lost production by increasing production after load reduction. Compared to the base case without interruptible load, the total cost reduces by $1.8 \%$, which may seem small but is actually significant at such industrial scale.

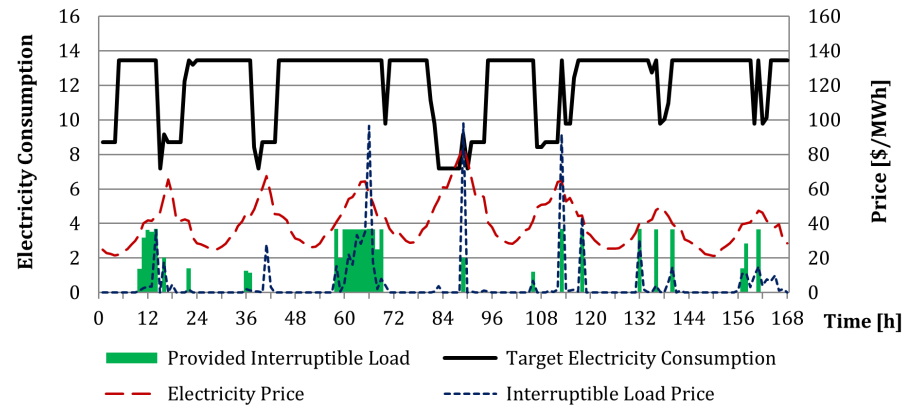

Figure 10: Target electricity consumption profile and provided interruptible load for the case of $\bar{\zeta}=23$, and price profiles. 

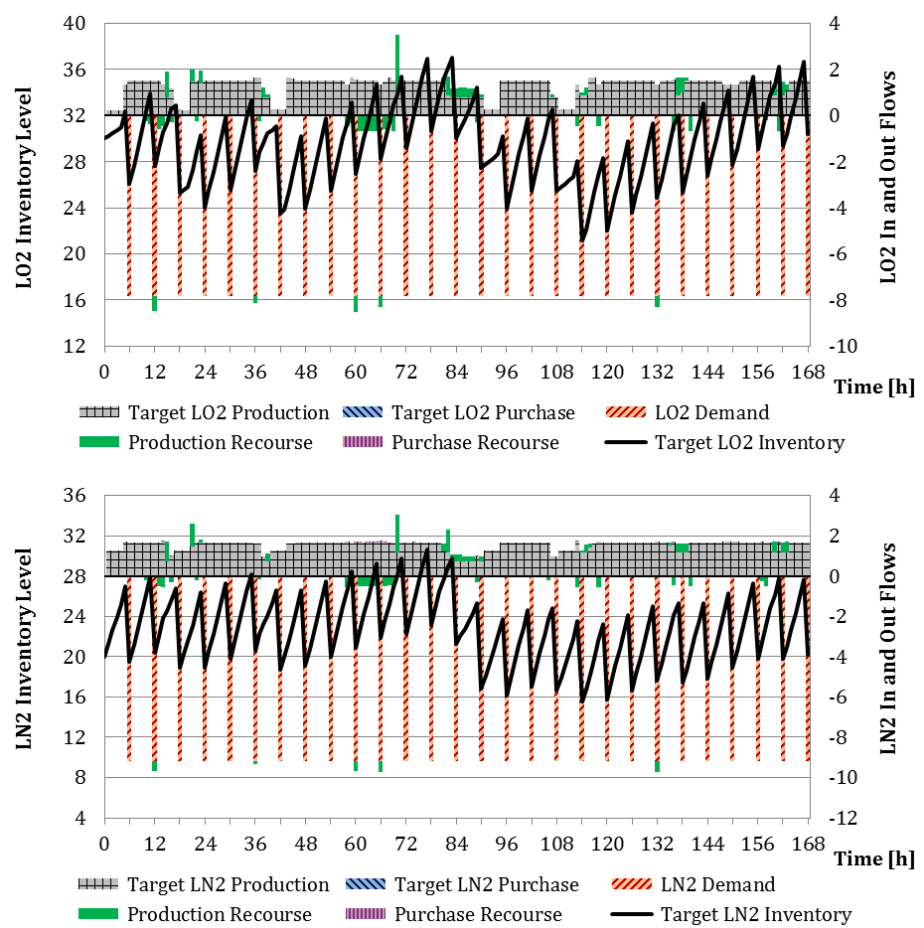

Figure 11: Target and recourse product flows and target inventory profile for the case of $\bar{\zeta}=23$.

Figure 12 shows the relationship between cost savings and level of plant utilization. Similar to the illustrative example, the cost reduction compared to the solution without interruptible load reaches its maximum at approximately $95 \%$ plant utilization; it drops to zero at $100 \%$ since production at full capacity is needed to satisfy demand, and the cost of purchasing additional products as recourse outweighs the benefit of providing interruptible load.

\section{Conclusions}

This work has addressed the robust scheduling of power-intensive industrial processes that can participate in the operating reserve market by providing interruptible load. An adjustable robust optimization approach has been applied to formulate a discrete-time MILP model that accounts for the uncertainty in load reduction demand, while considering recourse actions in the form of linear decision rules. The solution of the proposed model is guaranteed to be robust with respect to a budget uncertainty set that can be adjusted to change the level of conservatism.

An illustrative example and a real-world industrial air separation case demonstrate the capability of the proposed model. The case studies show that signifi- 


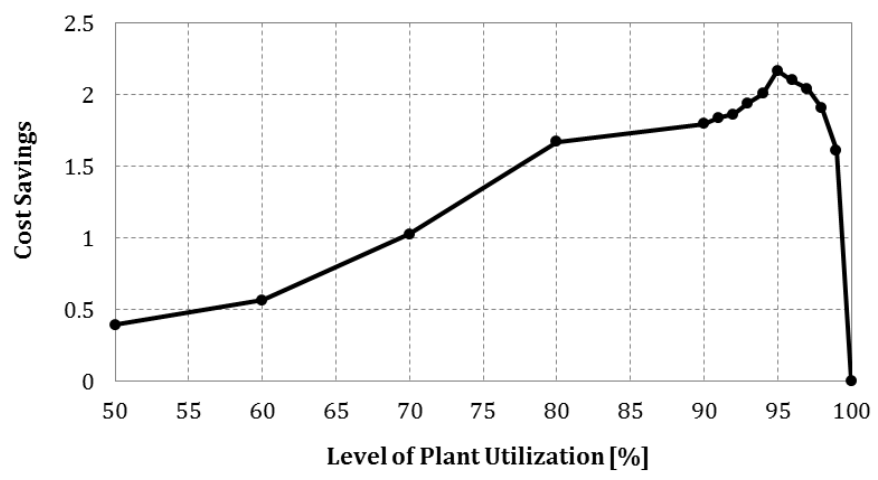

Figure 12: Absolute cost savings vs. level of plant utilization in the industrial case study.

cant financial benefits can be achieved by selling interruptible load. The results further demonstrate the value of recourse as the cost savings increase with the extent of recourse, in this case namely the number of uncertain parameters in preceding time periods considered in the decision rules. However, this flexibility in the recourse comes at the cost of increased model size and computation time. This trade-off has to be carefully considered in large-scale problems.

Moreover, contrary to results in the literature indicating that DR is more effective in plants with lower utilization, we find that this is not true when interruptible load is provided. Here, the largest cost savings are achieved at a high, yet not maximum level of plant utilization. The explanation is that higher plant utilization allows larger amount of interruptible load to be provided, yet some flexibility is still required for the implementation of effective recourse.

\section{Nomenclature}

Indices

$\begin{array}{ll}f & \text { supporting hyperplanes } \\ i & \text { products } \\ j & \text { vertices } \\ m, m^{\prime}, m^{\prime \prime} & \text { operating modes } \\ r & \text { operating subregions } \\ t & \text { time periods }\end{array}$

Sets

$F_{m r} \quad$ supporting hyperplanes associated with subregion $r$ of mode $m$

I products

$J_{m r} \quad$ vertices of polytope describing subregion $r$ of mode $m$ 


$\begin{array}{ll}\frac{M}{M} & \begin{array}{l}\text { operating modes } \\ \text { flexible operating modes, } \bar{M} \subseteq M\end{array} \\ \widehat{M} & \text { inflexible operating modes, } \widehat{M} \subseteq M \\ R_{m} & \text { subregions of mode } m \\ S Q & \text { predefined sequences of mode transitions } \\ T & \text { time periods, } T=\left\{-\theta^{\max }+1,-\theta^{\max }+2, \ldots, 0,1, \ldots, t^{\mathrm{fin}}\right\} \\ \bar{T} & \text { time period in the scheduling horizon, } \bar{T}=\left\{1,2, \ldots, t^{\mathrm{fin}}\right\} \\ T R & \text { possible mode transitions } \\ \overline{T R}_{m} & \text { modes from which mode } m \text { can be directly reached } \\ \widehat{T R}_{m} & \text { modes which can be directly reached from mode } m\end{array}$

Deterministic parameters

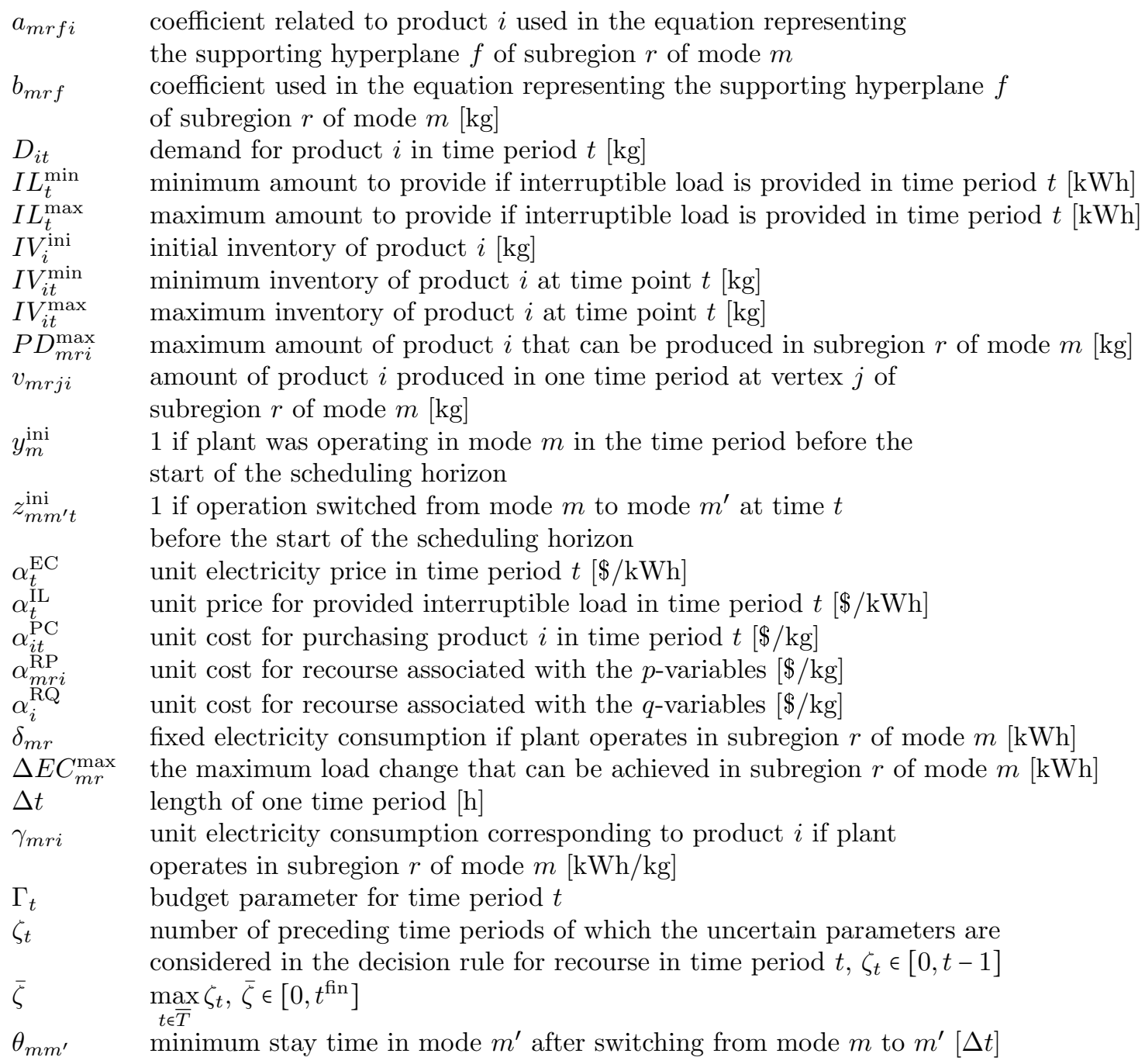


$\bar{\theta}_{m m^{\prime} m^{\prime \prime}}$

$\theta^{\max }$

$\Omega$ fixed stay time in mode $m^{\prime}$ of the predefined sequence $\left(m, m^{\prime}, m^{\prime \prime}\right)[\Delta t]$ maximum minimum or predefined stay time in a mode $[\Delta t]$

big-M parameter $[\mathrm{kWh}]$

Uncertain parameters

$L R_{t} \quad$ amount of load reduction requested in time period $t$

$w_{t} \quad$ normalized load reduction request in time period $t$

\section{Continuous variables}

$E C_{t} \quad$ amount of electricity consumed in time period $t[\mathrm{kWh}]$

$I L_{t} \quad$ amount of interruptible load provided in time period $t[\mathrm{kWh}]$

$I V_{i t} \quad$ inventory of product $i$ at time $t[\mathrm{~kg}]$

$p_{\text {mritk }} \quad$ coefficient for recourse decision rule related to the change in the amount of product $i$ produced in subregion $r$ of mode $m$ in time period $t$ in response to uncertainty realized in time period $k[\mathrm{~kg}]$

$P C_{i t} \quad$ amount of product $i$ purchased in time period $t[\mathrm{~kg}]$

$\widehat{P C}_{i t} \quad$ target amount of product $i$ purchased in time period $t[\mathrm{~kg}]$

$\widetilde{P C}_{i t} \quad$ response change in amount of product $i$ purchased in time period $t[\mathrm{~kg}]$

$P D_{i t} \quad$ amount of product $i$ produced in time period $t[\mathrm{~kg}]$

$q_{i t k} \quad$ coefficient for recourse decision rule related to the change in the amount of product $i$ purchased in time period $t$ in response to uncertainty realized in time period $k[\mathrm{~kg}]$

$\overline{P D}_{\text {mrit }} \quad$ amount of product $i$ produced in subregion $r$ of mode $m$ in time period $t[\mathrm{~kg}]$

$\widehat{P D}_{\text {mrit }} \quad$ target amount of product $i$ produced in subregion $r$ of mode $m$ in time period $t[\mathrm{~kg}]$

$\widetilde{P D}_{\text {mrit }} \quad$ response change in amount of product $i$ produced in subregion $r$ of mode $m$ in time period $t[\mathrm{~kg}]$

$S L_{i t} \quad$ amount of product $i$ sold in time period $t[\mathrm{~kg}]$

$T C \quad$ total net operating cost [\$]

$\lambda_{m r j t} \quad$ coefficient for vertex $j$ in subregion $r$ of mode $m$ in time period $t$

Binary variables

$x_{t} \quad 1$ if interruptible load is provided in time period $t$

$y_{m t} \quad 1$ if plant operates in mode $m$ in time period $t$

(can also be defined as a continuous variable)

$\bar{y}_{m r t} \quad 1$ if plant operates in subregion $r$ of mode $m$ in time period $t$

$z_{m m^{\prime} t} 1$ if plant operation switched from mode $m$ to mode $m^{\prime}$ at time $t$

\section{Acknowledgements}

The authors gratefully acknowledge the financial support from the National Science Foundation under Grant No. 1159443 and from Praxair. 


\section{Appendix A. Derivation of the ARC}

To each constraint in Problem (17), we apply the worst-case approach with respect to the uncertainty. As a result, the following bilevel problem is obtained:

$$
\begin{aligned}
\min \sum_{t \in \bar{T}}\left[\sum_{m} \sum_{r \in R_{m}} \alpha_{t}^{\mathrm{EC}}\left(\delta_{m r} \bar{y}_{m r t}+\sum_{i} \gamma_{m r i} \widehat{P D}_{m r i t}\right)+\sum_{i} \alpha_{i t}^{\mathrm{PC}} \widehat{P C}_{i t}-\alpha_{t}^{\mathrm{IL}} I L_{t}\right. \\
\left.+\sum_{m} \sum_{r \in R_{m}} \sum_{i} \alpha_{m r i}^{\mathrm{RP}}\left(-p_{m r i t t}+\sum_{k=t-\zeta_{t}}^{t-1} p_{m r i t k}\right)+\sum_{i} \sum_{k=t-\zeta_{t}}^{t} \alpha_{i}^{\mathrm{RQ}} q_{i t k}\right] \\
+\max _{w \in W(I L)}\left\{\sum_{t \in \bar{T}}\left(\sum_{m} \sum_{r \in R_{m}} \sum_{i} \sum_{k=t-\zeta_{t}}^{t} \alpha_{t}^{\mathrm{EC}} \gamma_{m r i} p_{m r i t k} w_{k}+\sum_{i} \sum_{k=t-\zeta_{t}}^{t} \alpha_{i t}^{\mathrm{PC}} q_{i t k} w_{k}\right)\right\}
\end{aligned}
$$

s.t. $\quad$ Eqs. (1e)-(1f), (2)-(4), (8), (9b)-(9c), (15c)-(15e), (17f)-(17k)

$$
\begin{aligned}
& \sum_{i} a_{m r f i} \widehat{P D}_{m r i t}+\min _{w \in W(I L)}\left\{\sum_{i} a_{m r f i} \sum_{k=t-\zeta_{t}}^{t} p_{m r i t k} w_{k}\right\} \geq b_{m r f} \bar{y}_{m r t} \quad \forall m, r \in R_{m}, f \in F_{m r}, t \in \bar{T} \\
& \widehat{P D}_{m r i t}+\max _{w \in W(I L)}\left\{\sum_{k=t-\zeta_{t}}^{t} p_{m r i t k} w_{k}\right\} \leq P D_{m r i}^{\max } \bar{y}_{m r t} \quad \forall m, r \in R_{m}, i, t \in \bar{T} \\
& \widehat{P D}_{m r i t}+\min _{w \in W(I L)}\left\{\sum_{k=t-\zeta_{t}}^{t} p_{m r i t k} w_{k}\right\} \geq 0 \quad \forall m, r \in R_{m}, i, t \in \bar{T} \quad \text { (A.1d) } \\
& I V_{i, 0}+\sum_{k=1}^{t}\left(\sum_{m} \sum_{r \in R_{m}} \widehat{P D}_{m r i k}+\widehat{P C}_{i k}-D_{i k}\right) \\
& +\max _{w \in W(I L)}\left\{\sum_{k=1}^{t}\left(\sum_{m} \sum_{r \in R_{m}} \sum_{l=k-\zeta_{k}}^{k} p_{m r i k l} w_{l}+\sum_{l=k-\zeta_{k}}^{k} q_{i k l} w_{l}\right)\right\} \leq I V_{i}^{\max } \quad \forall i, t \in \bar{T} \\
& I V_{i, 0}+\sum_{k=1}^{t}\left(\sum_{m} \sum_{r \in R_{m}} \widehat{P D}_{m r i k}+\widehat{P C}_{i k}-D_{i k}\right) \\
& +\min _{w \in W(I L)}\left\{\sum_{k=1}^{t}\left(\sum_{m} \sum_{r \in R_{m}} \sum_{l=k-\zeta_{k}}^{k} p_{m r i k l} w_{l}+\sum_{l=k-\zeta_{k}}^{k} q_{i k l} w_{l}\right)\right\} \geq I V_{i}^{\min } \quad \forall i, t \in \bar{T} \\
& \max _{w \in W(I L)}\left\{\sum_{m} \sum_{r \in R_{m}} \sum_{i} \sum_{k=t-\zeta_{t}}^{t} \gamma_{m r i} p_{m r i t k} w_{k}+I L_{t} w_{t}\right\} \leq \Omega\left(1-x_{t}\right) \quad \forall t \in \bar{T}
\end{aligned}
$$

Tables A.12 and A.13 list the lower-level problems and the corresponding 
dual formulations, respectively. Due to strong duality, the bilevel problem can be reformulated into a single-level problem by substituting the dual formulations into Eqs. (A.1), which yields the ARC given by Eqs. (19).

Table A.12: List of lower-level problems

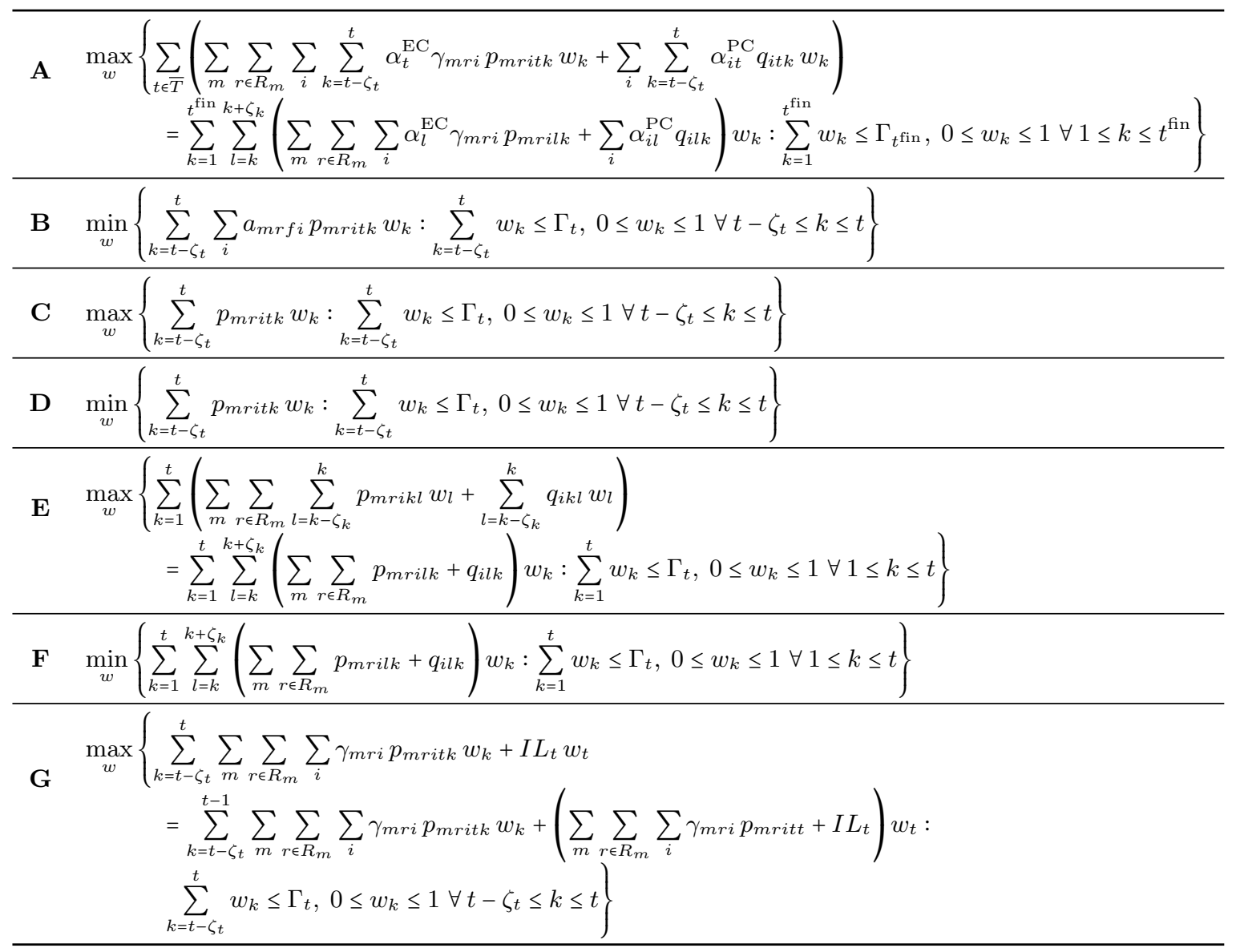


Table A.13: Dual formulations of lower-level problems

\begin{tabular}{|c|c|c|}
\hline & $\min _{u^{\mathrm{A}}, s^{\mathrm{A}}}\{$ & $\begin{array}{c}\left\{\Gamma_{t^{\mathrm{fin}}} u^{\mathrm{A}}+\sum_{k=1}^{t^{\mathrm{fin}}} s_{k}^{\mathrm{A}}: u^{\mathrm{A}}+s_{k}^{\mathrm{A}} \geq \sum_{l=k}^{k+\zeta_{k}} \sum_{i}\left(\sum_{m} \sum_{r \in R_{m}} \alpha_{l}^{\mathrm{EC}} \gamma_{m r i} p_{m r i l k}+\alpha_{i l}^{\mathrm{PC}} q_{i l k}\right) \forall 1 \leq k \leq t^{\mathrm{fin}},\right. \\
\left.u^{\mathrm{A}} \geq 0, s_{k}^{\mathrm{A}} \geq 0 \forall 1 \leq k \leq t^{\mathrm{fin}}\right\}\end{array}$ \\
\hline $\mathbf{A}$ & $\max _{u^{\mathrm{B}}, s^{\mathrm{B}}}$ & $\begin{array}{c}\Gamma_{t} u_{m r f t}^{\mathrm{B}}+\sum_{k=t-\zeta_{t}}^{t} s_{m r f t k}^{\mathrm{B}}: u_{m r f t}^{\mathrm{B}}+s_{m r f t k}^{\mathrm{B}} \leq \sum_{i} a_{m r f i} p_{m r i t k} \forall t-\zeta_{t} \leq k \leq t, \\
\left.u_{m r f t}^{\mathrm{B}} \leq 0, s_{m r f t k}^{\mathrm{B}} \leq 0 \forall t-\zeta_{t} \leq k \leq t\right\}\end{array}$ \\
\hline C & $\min _{u^{\mathrm{C}}, s^{\mathrm{C}}}$ & $\left\{\Gamma_{t} u_{m r i t}^{\mathrm{C}}+\sum_{k=t-\zeta_{t}}^{t} s_{m r i t k}^{\mathrm{C}}: u_{m r i t}^{\mathrm{C}}+s_{m r i t k}^{\mathrm{C}} \geq p_{m r i t k} \forall t-\zeta_{t} \leq k \leq t, u_{m r i t}^{\mathrm{C}} \geq 0, s_{m r i t k}^{\mathrm{C}} \geq 0 \forall t-\zeta_{t} \leq k \leq t\right\}$ \\
\hline D & $\max _{u^{\mathrm{D}}, s^{\mathrm{D}}}$ & $\left\{\Gamma_{t} u_{m r i t}^{\mathrm{D}}+\sum_{k=t-\zeta_{t}}^{t} s_{m r i t k}^{\mathrm{D}}: u_{m r i t}^{\mathrm{D}}+s_{m r i t k}^{\mathrm{D}} \leq p_{m r i t k} \forall t-\zeta_{t} \leq k \leq t, u_{m r i t}^{\mathrm{D}} \leq 0, s_{m r i t k}^{\mathrm{D}} \leq 0 \forall t-\zeta_{t} \leq k \leq t\right\}$ \\
\hline $\mathbf{E}$ & $\min _{u^{\mathrm{E}}, s^{\mathrm{E}}}$ & $\left\{\Gamma_{t} u_{i t}^{\mathrm{E}}+\sum_{k=1}^{t} s_{i t k}^{\mathrm{E}}: u_{i t}^{\mathrm{E}}+s_{i t k}^{\mathrm{E}} \geq \sum_{l=k}^{k+\zeta_{k}}\left(\sum_{m} \sum_{r \in R_{m}} p_{m r i l k}+q_{i l k}\right) \forall 1 \leq k \leq t, u_{i t}^{\mathrm{E}} \geq 0, s_{i t k}^{\mathrm{E}} \geq 0 \forall 1 \leq k \leq t\right\}$ \\
\hline $\mathbf{F}$ & $\max _{u^{\mathrm{F}}, s^{\mathrm{F}}}$ & $\left\{\Gamma_{t} u_{i t}^{\mathrm{F}}+\sum_{k=1}^{t} s_{i t k}^{\mathrm{F}}: u_{i t}^{\mathrm{F}}+s_{i t k}^{\mathrm{F}} \leq \sum_{l=k}^{k+\zeta_{k}}\left(\sum_{m} \sum_{r \in R_{m}} p_{m r i l k}+q_{i l k}\right) \forall 1 \leq k \leq t, u_{i t}^{\mathrm{F}} \leq 0, s_{i t k}^{\mathrm{F}} \leq 0 \forall 1 \leq k \leq t\right\}$ \\
\hline $\mathbf{G}$ & $\min _{u^{G}, s^{G}}$ & $\begin{aligned}\left\{\Gamma_{t} u_{t}^{\mathrm{G}}+\sum_{k=t-\zeta t}^{t} s_{t k}^{\mathrm{G}}: u_{t}^{\mathrm{G}}+s_{t k}^{\mathrm{G}} \geq \sum_{m} \sum_{r \in R_{m}} \sum_{i} \gamma_{m r i} p_{m r i t k} \forall t-\zeta_{t} \leq k \leq t-1,\right. \\
\left.u_{t}^{\mathrm{G}}+s_{t t}^{\mathrm{G}} \geq \sum_{m} \sum_{r \in R_{m}} \sum_{i} \gamma_{m r i} p_{m r i t t}+I L_{t}, u_{t}^{\mathrm{G}} \geq 0, s_{t k}^{\mathrm{G}} \geq 0 \forall t-\zeta_{t} \leq k \leq t\right\}\end{aligned}$ \\
\hline
\end{tabular}

\section{References}

Aalami, H. A., Moghaddam, M. P., Yousefi, G. R., 2010. Demand response modeling considering Interruptible/Curtailable loads and capacity market programs. Applied Energy 87 (1), 243-250.

Aminifar, F., Fotuhi-Firuzabad, M., Shahidehpour, M., 2009. Unit commitment with probabilistic spinning reserve and interruptible load considerations. IEEE Transactions on Power Systems 24 (1), 388-397.

Ashok, S., 2006. Peak-load management in steel plants. Applied Energy 83 (5), 413-424.

Babu, C. A., Ashok, S., 2008. Peak Load Management in Electrolytic Process Industries. IEEE Transactions on Power Systems 23 (2), 399-405.

Bai, J., Gooi, H. B., Xia, L. M., Strbac, G., Venkatesh, B., 2006. A Probabilistic Reserve Market Incorporating Interruptible Load. IEEE Transactions on Power Systems 21 (3), 1079-1087. 
Ben-Tal, A., Chung, B. D., Mandala, S. R., Yao, T., 2011. Robust optimization for emergency logistics planning: Risk mitigation in humanitarian relief supply chains. Transportation Research Part B: Methodological 45 (8), 11771189.

Ben-Tal, A., El Ghaoui, L., Nemirovski, A., 2009. Robust Optimization. Princeton University Press, New Jersey.

Ben-Tal, A., Goryashko, A., Guslitzer, E., Nemirovski, A., 2004. Adjustable robust solutions of uncertain linear programs. Mathematical Programming 99 (2), 351-376.

Bertsimas, D., Sim, M., 2004. The Price of Robustness. Operations Research $52(1), 35-53$.

Castro, P. M., Harjunkoski, I., Grossmann, I. E., 2011. Optimal scheduling of continuous plants with energy constraints. Computers \& Chemical Engineering 35 (2), 372-387.

Castro, P. M., Sun, L., Harjunkoski, I., 2013. Resource-Task Network Formulations for Industrial Demand Side Management of a Steel Plant. Industrial \& Engineering Chemistry Research 52, 13046-13058.

Chen, X., Zhang, Y., 2009. Uncertain Linear Programs: Extended Affinely Adjustable Robust Counterparts. Operations Research 57 (6), 1469-1482.

EnerNOC, 2014. PJM's Synchronized Reserve Market. URL http://www.enernoc.com/our-resources/brochures-faq

FERC, 2010. National Action Plan on Demand Response. Tech. rep., Federal Energy Regulatory Commission.

Fotuhi-Firuzabad, M., Billinton, R., 2000. Impact of load management on composite system reliability evaluation short-term operating benefits. IEEE Transactions on Power Systems 15 (2), 858-864.

GAMS Development Corporation, 2015. GAMS version 24.4.1.

Hatami, A. R., Seifi, H., Sheikh-El-Eslami, M. K., 2009. Hedging risks with interruptible load programs for a load serving entity. Decision Support Systems 48 (1), 150-157.

Ierapetritou, M. G., Wu, D., Vin, J., Sweeney, P., Chigirinskiy, M., 2002. Cost Minimization in an Energy-Intensive Plant Using Mathematical Programming Approaches. Industrial \& Engineering Chemistry Research 41 (21), 52625277 .

Karwan, M. H., Keblis, M. F., 2007. Operations planning with real time pricing of a primary input. Computers \& Operations Research 34 (3), 848-867. 
Kuhn, D., Wiesemann, W., Georghiou, A., 2011. Primal and dual linear decision rules in stochastic and robust optimization. Mathematical Programming 130 (1), 177-209.

Merkert, L., Harjunkoski, I., Isaksson, A., Säynevirta, S., Saarela, A., Sand, G., 2014. Scheduling and energy - Industrial challenges and opportunities. Computers \& Chemical Engineering 72, 183-198.

Mitra, S., Grossmann, I. E., Pinto, J. M., Arora, N., 2012. Optimal production planning under time-sensitive electricity prices for continuous power-intensive processes. Computers \& Chemical Engineering 38, 171-184.

Mitra, S., Sun, L., Grossmann, I. E., 2013. Optimal scheduling of industrial combined heat and power plants under time-sensitive electricity prices. Energy 54, 194-211.

Paulus, M., Borggrefe, F., 2011. The potential of demand-side management in energy-intensive industries for electricity markets in Germany. Applied Energy 88 (2), 432-441.

PJM Interconnection LLC, 2013a. Day-Ahead LMP Data.

URL http://www.pjm.com/markets-and-operations/energy/day-ahead/ lmpda.aspx

PJM Interconnection LLC, 2013b. Preliminary Billing Reports - Ancillary Services Market Data.

URL http://www.pjm.com/markets-and-operations/ market-settlements/preliminary-billing-reports.aspx

Samad, T., Kiliccote, S., 2012. Smart grid technologies and applications for the industrial sector. Computers \& Chemical Engineering 47, 76-84.

Sung, C., Maravelias, C. T., 2009. A Projection-Based Method for Production Planning of Multiproduct Facilities. AIChE Journal 55 (10), 2614-2630.

Tuan, L. A., Bhattacharya, K., 2003. Competitive framework for procurement of interruptible load services. IEEE Transactions on Power Systems 18 (2), 889-897.

Vujanic, R., Mariéthos, S., Goulart, P., Morari, M., 2012. Robust Integer Optimization and Scheduling Problems for Large Electricity Consumers. In: Proceedings of the 2012 American Control Conference. pp. 3108-3113.

Zhang, Q., Grossmann, I. E., 2015. Planning and Scheduling for Industrial Demand Side Management: Advances and Challenges. To appear in Alternative Energy Sources and Technologies: Process Design and Operation.

Zhang, Q., Grossmann, I. E., Heuberger, C. F., Sundaramoorthy, A., Pinto, J. M., 2015a. Air Separation with Cryogenic Energy Storage: Optimal Scheduling Considering Electric Energy and Reserve Markets. AIChE Journal 61 (5), 1547-1558. 
Zhang, Q., Grossmann, I. E., Sundaramoorthy, A., Pinto, J. M., 2015b. Datadriven construction of Convex Region Surrogate models. Optimization and Engineering.

Zhang, Q., Sundaramoorthy, A., Grossmann, I. E., Pinto, J. M., 2016. A discrete-time scheduling model for continuous power-intensive process networks with various power contracts. Computers \& Chemical Engineering 84, 382-393. 